\title{
Interloper treatment in dynamical modelling of galaxy clusters
}

\author{
R. Wojtak ${ }^{1}$, E. L. Łokas $^{1}$, G. A. Mamon²,3, S. Gottlöber ${ }^{4}$, F. Prada ${ }^{5}$, and M. Moles ${ }^{5}$ \\ 1 Nicolaus Copernicus Astronomical Center, Bartycka 18, 00-716 Warsaw, Poland \\ e-mail: wojtak@camk.edu.pl \\ 2 Institut d'Astrophysique de Paris (UMR 7095: CNRS and Université Pierre \& Marie Curie), 98 bis Bd Arago, 75014 Paris, France \\ GEPI (UMR 8111: CNRS and Université Denis Diderot), Observatoire de Paris, 92195 Meudon, France \\ Astrophysikalisches Institut Potsdam, An der Sternwarte 16, 14482 Potsdam, Germany \\ 5 Instituto de Astrofísica de Andalucia (CSIC), Apartado Correos 3005, 18080 Granada, Spain
}

Received 24 November 2006 / Accepted 1 February 2007

\section{ABSTRACT}

\begin{abstract}
Aims. The aim of this paper is to study the efficiency of different approaches to interloper treatment in dynamical modelling of galaxy clusters.

Methods. Using cosmological $N$-body simulation of standard $\Lambda$ CDM model, we select 10 massive dark matter haloes and use their particles to emulate mock kinematic data in terms of projected galaxy positions and velocities as they would be measured by a distant observer. Taking advantage of the full 3D information available from the simulation, we select samples of interlopers defined with different criteria. The interlopers thus selected provide means to assess the efficiency of different interloper removal schemes found in the literature.

Results. We study direct methods of interloper removal based on dynamical or statistical restrictions imposed on ranges of positions and velocities available to cluster members. In determining these ranges, we use either the velocity dispersion criterion or a maximum velocity profile. We also generalize the common approaches taking into account both the position and velocity information. Another criterion is based on the dependence of the commonly used virial mass and projected mass estimators on the presence of interlopers. We find that the direct methods exclude on average 60-70 percent of unbound particles producing a sample with contamination as low as 2-4 percent. Next, we consider indirect methods of interloper treatment which are applied to the data stacked from many objects. In these approaches, interlopers are treated in a statistical way as a uniform background which modifies the distribution of cluster members. Using a Bayesian approach, we reproduce the properties of composite clusters and estimate the probability of finding an interloper as a function of distance from the object centre.
\end{abstract}

Key words. galaxies: clusters: general - cosmology: dark matter - methods: $N$-body simulations - methods: numerical methods: statistical

\section{Introduction}

The modelling of galaxy kinematics in clusters remains one of the major tools in determining their properties, in particular their mass distribution and dark matter content. Due to projection effects, any cluster kinematic data sample inevitably contains galaxies that are not bound to the cluster and therefore are not good tracers of its gravitational potential. We will call these galaxies interlopers. An essential step in dynamical modelling of clusters by any method is therefore to remove such interlopers from the samples or take their presence into account statistically. Velocity information can be used to remove obvious interlopers that are thousands of $\mathrm{km} \mathrm{s}^{-1}$ off the mean cluster velocity, but there remain numerous interlopers that lie in a similar general velocity range as the cluster members. Some hints can be provided by studying the photometric properties of galaxies or restricting the samples to elliptical galaxies but these approaches usually do not solve the problem completely.

It has long been recognized that the line-of-sight velocity distribution of galaxies in clusters is close to a Gaussian. The first attempts to design a scheme to remove the interlopers were based on this property. Yahil \& Vidal (1977) proposed to calculate the line-of-sight velocity dispersion of the galaxy sample, $\sigma_{\text {los }}$, and iteratively remove outliers with velocities larger than $3 \sigma_{\text {los. }}$. This simple approach is still widely used today. With enough galaxies in a sample, one can take into account the dependence of $\sigma_{\text {los }}$ on the projected distance from the cluster centre $R$ and perform the rejection procedure in bins with different $\sigma_{\text {los }}$ or fit a simple solution of the Jeans equation to the measured line-of-sight velocity dispersion profile, $\sigma_{\text {los }}(R)$, and reject galaxies outside the $3 \sigma_{\text {los }}(R)$ curves (Łokas et al. 2006).

Perea et al. (1990) discussed another method relying on iterative removal of galaxies whose absence in the sample causes the biggest change in the mass estimator. Zabludoff et al. (1990), Katgert et al. (1996) and Fadda et al. (1996) advertised the use of gaps in the velocity distribution as a way to separate interlopers from real cluster members. Diaferio \& Geller (1997) and Diaferio (1999) proposed the use of caustics where the projected distribution function is sufficiently low to separate cluster members from the surrounding medium. Prada et al. (2003) discussed the solution to the problem based on the use of escape velocities. The first methods that combine the information on the position and velocity of a galaxy were proposed by den Hartog \& Katgert (1996) and Fadda et al. (1996). All these methods aim at cleaning the galaxy sample from non-members before attempting the proper dynamical analysis of the cluster; we call them direct methods of interloper removal.

A completely new approach to interloper treatment was pioneered by van der Marel et al. (2000) where, for the first time, the interlopers were not identified and removed from the sample, 
but their presence was taken into account statistically by appropriate modification of the distribution function of the galaxies. A similar approach was also considered by Mahdavi \& Geller (2004) with more realistic assumptions concerning the distribution of interlopers. Prada et al. (2003) studied the distribution of satellites around giant galaxies by fitting to the projected velocity distribution the sum of a Gaussian and a uniform distribution taking care of the background. We will refer to this type of methods as indirect. It should be noted that these methods are mainly applicable to composite clusters, i.e. data sets created by combining kinematic data from many objects because only then the samples are numerous enough to provide useful constraints on the interloper fraction.

The different methods of interloper treatment found in the literature are difficult to compare. Each one of them has a different set of underlying assumptions. They also differ by the amount of parameters that have to be put in by hand. Most of the methods are iterative and some may not converge. The ultimate comparison between the methods can only be performed by resorting to $N$-body simulations where full $3 \mathrm{D}$ information is available and true interlopers can be identified. Such tests have been already attempted (e.g. by Perea et al. 1990; den Hartog \& Katgert 1996; Diaferio 1999). In particular, van Haarlem et al. (1997) compared the methods of den Hartog \& Katgert (1996) and Yahil \& Vidal (1977) in terms of the quality of reproduction of the real velocity dispersion. However, more systematic study of different procedures is still needed and this is the aim of the present paper. We implement and generalize different prescriptions for interloper removal found in the literature and apply them to mock kinematic data created from the simulation. Our goal is to measure the efficiency of the different methods by measuring fractions of interlopers they remove.

Our choice of methods will of course be arbitrary. We tried to focus on those easiest to implement, most widely used in the literature and with the smallest number of pre-selected parameters so that they are applicable not only to galaxy clusters but to all astronomical systems where kinematic measurements of discrete tracer can be made (e.g. dwarf spheroidal galaxies). In the near future we plan to apply the methods discussed here to nearby clusters from the WINGS survey (Fasano et al. 2006) where about 300 redshifts per cluster will be available.

The problem of the treatment of interlopers is directly related to the problem of the mass estimation in gravitationally bound objects. We will demonstrate in Sect. 2 that using contaminated kinematic samples can lead to serious errors in the estimated mass. In addition, several of the interloper removal schemes we discuss make use of some crude mass estimators. However, the purpose of this work is not to provide the best method for mass estimation in galaxy clusters. Instead, we focus on a much narrower issue of how to obtain a clean sample of cluster galaxies free of interlopers before attempting a further analysis of the mass distribution in the cluster. This final analysis can be performed via a number of methods e.g. fitting velocity dispersion profile assuming isotropic orbits (e.g. Biviano \& Girardi 2003), fitting velocity dispersion and kurtosis for arbitrary constant anisotropy (Łokas et al. 2006) etc. The final outcome of these procedures will depend on their specific properties and on the properties of objects to which they are applied (e.g. whether they are spherically symmetric, depart from equilibrium, how well they are sampled etc.). For example, Sanchis et al. (2004) and Łokas et al. (2006) applied the dispersion+kurtosis fitting method to simulated clusters (after removal of interlopers) and discussed how well the main properties of the clusters (including the mass) are reproduced.
For the purpose of this study we used a present-day output of a pure dark matter, medium-resolution cosmological $N$-body simulation in which cluster-size haloes can be identified. Taking advantage of the fact that the distribution of galaxies in clusters is similar to mass distribution in simulated dark matter haloes, i.e. both are cuspy and can be approximated by the NFW (Navarro et al. 1997) profile (e.g. Carlberg et al. 1997; Łokas \& Mamon 2003; Biviano \& Girardi 2003) we assumed that the galaxies can be approximated by just dark matter particles. Although it would be worthwhile to test the methods on a set of higher resolution simulations where galaxies or subhaloes can be identified, the distributions of subhaloes both in space and velocity are known to be biased with respect to those of dark matter particles (Diemand et al. 2004). On the other hand, Faltenbacher \& Diemand (2006) have recently shown that subhaloes with sufficiently high mass corresponding to galaxies have distributions much less biased and very similar to those of dark matter particles, which makes the effort of using subhaloes questionable. Nevertheless, a possibility to assign stellar populations to subhaloes can considerably improve their usefulness in the analysis. As shown by Biviano et al. (2006), subhaloes with old stellar populations are more concentrated around their mother haloes so by selecting them one can reduce the contamination by interlopers.

The paper is organized as follows. In Sect. 2 we describe the way to create mock data sets from the simulations, introduce different types of interlopers and discuss how the presence of interlopers can affect the inferred properties of a galaxy cluster. Sect. 3 is devoted to direct methods of interloper removal. We first discuss the dynamical approach where the maximum velocity available to a member galaxy is estimated using some assumptions about the cluster mass profile. Next we study the statistical approach in its most commonly used forms which we then generalize by considering the distribution of galaxies in projected phase space. We also discuss the efficiency of different mass estimators in identifying interlopers. Section 4 is devoted to indirect methods of interloper treatment and the discussion follows in Sect. 5.

\section{Interlopers on velocity diagrams of simulated haloes}

In this work we used an $N$-body cosmological simulation of standard $\Lambda$ CDM model described in Wojtak et al. (2005). The simulation was performed using a version of the ART (Adaptive Refinement Tree) code (Kravtsov et al. 1997) in a box of size $150 h^{-1} \mathrm{Mpc}$ with parameters $h=0.7, \Omega_{M}=0.3, \Omega_{\Lambda}=0.7$ and $\sigma_{8}=0.9$. From the whole sample of dark matter haloes formed in the final simulation output $(z=0)$ we choose 10 massive $\left(10^{14}-10^{15} M_{\odot}\right)$ and possibly relaxed ones i.e. without any obvious signatures of ongoing major mergers. They are listed and described in Wojtak et al. (2005). All of them are characterized by mildly radial particle orbits and their density profiles are well fitted up to the virial radius $r_{\mathrm{v}}$ by the NFW formula

$$
\frac{\rho}{\rho_{c, 0}}=\frac{\Delta_{c} c^{2} g(c)}{3\left(r / r_{\mathrm{v}}\right)\left(1+c\left(r / r_{\mathrm{v}}\right)\right)^{2}},
$$

where $g(c)=[\ln (1+c)-c /(1+c)]^{-1}, c$ is the concentration parameter, $\rho_{c, 0}$ is the critical density at present and $\Delta_{c}$ is a parameter defining virial mass in terms of overdensity with respect to the critical density. We assume $\Delta_{c}=101.9$ which is the value valid for the concordance $\Lambda$ CDM model with $\Omega_{\mathrm{m}}=0.3$ and $\Omega_{\Lambda}=0.7$ 

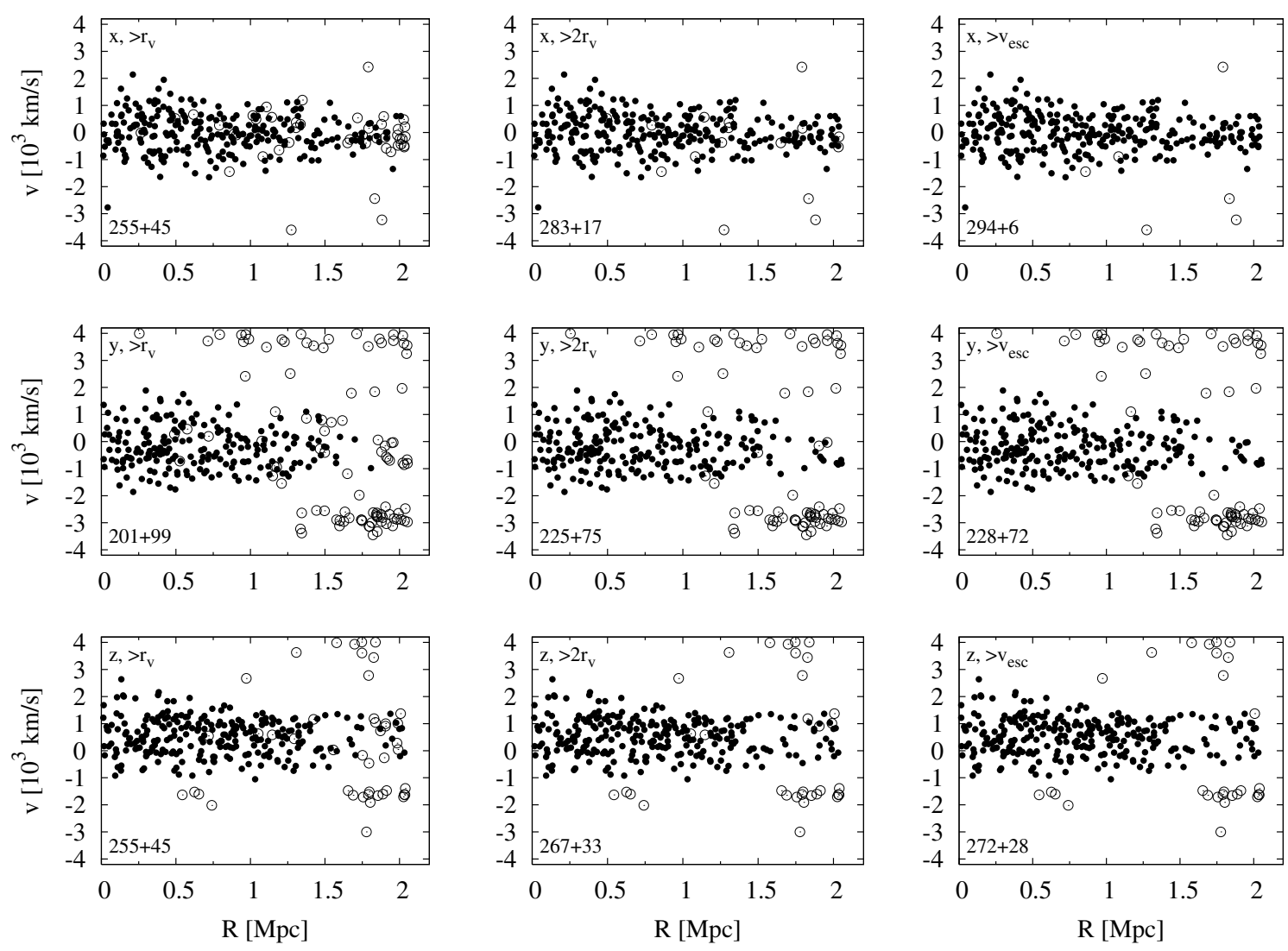

Fig. 1. Velocity diagrams of halo 6 out to $R=r_{\mathrm{v}}$ in the mass centre rest frame of reference seen in different projections and with different types of interlopers. Filled and empty circles indicate halo particles and interlopers respectively. In the top left corner of each panel we mark the projection axis and the criterion for interloper identification $\left(>r_{\mathrm{v}},>2 r_{\mathrm{v}}\right.$ or $>v_{\text {esc }}$ for particles beyond $r_{\mathrm{v}}, 2 r_{\mathrm{v}}$ and unbound particles respectively). In the bottom left corner we give numbers of halo particles and interlopers which are seen on a given velocity diagram.

(Łokas \& Hoffman 2001). The mean value of the concentration parameter averaged over all 10 haloes is equal to 7.2 .

In order to emulate kinematic data for a galaxy cluster embedded in a given dark mater halo we place an imaginary observer at the distance of $D=100 \mathrm{Mpc}$ from the halo centre (going from the simulation comoving coordinates to the observer's redshift space) so that the receding velocity of a halo mass centre observed by him is around $7000 \mathrm{~km} \mathrm{~s}^{-1}$. Approximating the conical shape of the observation beam with a cylinder $\left(D \gg r_{\mathrm{v}}\right)$, we project position vectors of simulation particles onto the plane perpendicular to the line of sight and their velocities with respect to the observer onto his line of sight. Assuming that some of the simulation particles represent galaxies, we randomly select 300 particles from the inside of the observation cylinder with projected radius $R=r_{\mathrm{v}}$, where the virial radius $r_{\mathrm{v}}$ is found in 3D analysis. Additionally, we restrict our selection to particles with velocities from the range $\pm 4000 \mathrm{~km} \mathrm{~s}^{-1}$ with respect to the velocity of a halo mass centre. This choice of velocity cut-off, corresponding to at least $4 \sigma_{\text {los }}$ for cluster-size objects, guarantees that we do not exclude any cluster galaxies with high peculiar velocities.

We place the cylinder of observation along the main axes of the simulation box so the orientation of the haloes (which have triaxial shapes) with respect to the line of sight should be random. Finally for each of the 10 haloes we obtain three sets of projected galaxy positions and velocities from observations along $x, y$ and $z$ axis of the simulation box. We will refer to these sets of data as velocity diagrams. Each velocity diagram includes both particles from the inside of a given halo (we call them simply halo particles) and particles from the outside of a halo which are seen because of the projection effects (we call them interlopers).

First we identify the true interlopers in our data using the full 3D information about positions and velocities of simulation particles. Obviously one can think about interlopers as particles which are beyond $r_{\mathrm{v}}$ since they are from the outside of virialized region and they are not used in the estimation of density profile. We find that on average 24 percent of particles on our velocity diagrams have $r>r_{\mathrm{v}}$. This criterion of interloper identification, however, seems to be too restrictive in many cases since the object may possess a virialized region or at least a well defined density profile extending up to radii beyond $r_{\mathrm{v}}$ defined by density contrast parameter $\Delta_{c}$ (e.g. Klypin et al. 2003; Wojtak et al. 2005; Prada et al. 2006). Besides, halo shapes are not spherical and imposing this kind of symmetry we can lose particles that are inside the real virialized region of the halo. We find that almost half of particles beyond $r_{\mathrm{v}}$ on our velocity diagrams reside below $\sim 2 r_{\mathrm{v}}$ and are bound to their halo (the fraction of unbound particles is negligible at $2 r_{\mathrm{v}}$ ). These considerations suggest that they could also be treated as good tracers of the halo potential. We have therefore decided to consider two more conservative criteria of interloper selection: particles beyond $2 r_{\mathrm{v}}$ and unbound particles (with velocity greater than the escape velocity). Average contribution of these groups to the particles on the velocity diagrams is 13 and 8 percent respectively. Figure 1 shows a set of velocity diagrams for halo 6 in different projections (rows) and with different criteria of interloper selection (columns), where filled and empty circles correspond to halo particles and interlopers respectively. 

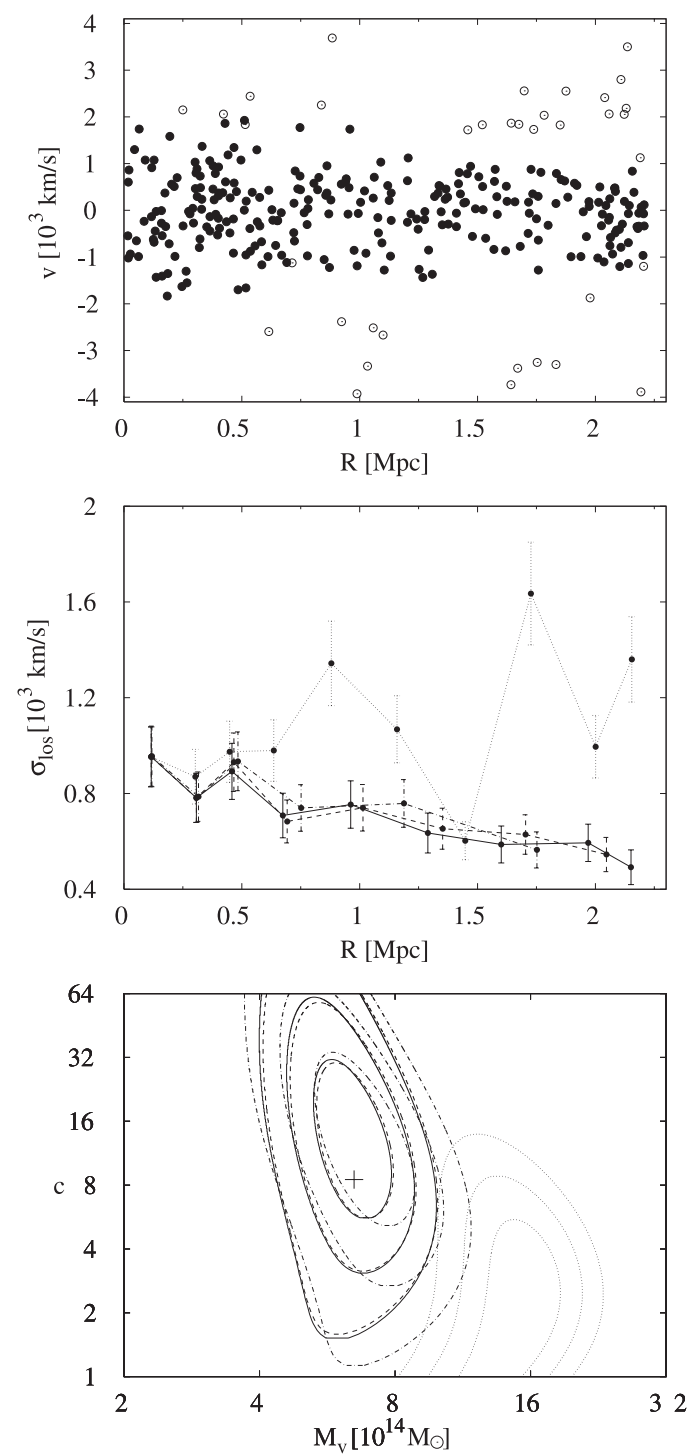

Fig. 2. The top panel shows the velocity diagram of halo 4 in projection along $z$ axis. Filled and empty circles indicate particles bound and unbound to this halo respectively. Middle and bottom panels show respectively the dispersion profiles and the results of the fitting procedure in the form of $68.3,95.4$ and 99.73 percent probability contours in the $M_{\mathrm{v}}-c$ parameter plane assuming isotropic orbits. The different types of lines correspond to different subsamples of particles used to calculate the dispersion profile: dotted lines are for the whole sample with interlopers included, solid ones for bound particles, dashed ones for particles below $2 r_{\mathrm{v}}$ and dotted-dashed lines for particles below $r_{\mathrm{v}}$. The cross marks the concentration parameter and virial mass found in $3 \mathrm{D}$ analysis of the mass distribution in the halo.

To illustrate how interlopers affect the results of dynamical analysis we fit for simplicity an isotropic solution of the Jeans equation to a velocity dispersion profile measured for one of the simulated velocity diagrams (see Łokas \& Mamon 2001, 2003 and Łokas et al. 2006 for details of the Jeans formalism and the fitting procedure). The dispersion profile is measured in radial bins for a whole sample (300 particles) and for three subsamples of particles cleaned of three types of interlopers introduced above. Figure 2 shows a typical velocity diagram generated from our halo 4 (with filled and empty circles as bound and unbound particles respectively), dispersion profiles for the four mentioned subsamples of particles and the corresponding results of the fitting procedure aimed at estimating the virial mass $M_{\mathrm{v}}$ and concentration $c$ of the halo, where parameter values found in $3 \mathrm{D}$ analysis are marked with a cross. All lines corresponding to the same particle sample are drawn with the lines of the same type.

Although the results shown in Fig. 2 concern just a single case of a velocity diagram, they illustrate well the general feature of bias caused by interlopers. First, note that the velocity dispersion is overestimated mainly in the outer part of the velocity diagram and this is caused mostly by unbound particles (since all dispersion profiles calculated for the data cleaned of interlopers of three different types, which include at least all unbound particles, are almost the same). Second, all three corrected dispersion profiles infer fitting results which are very similar to each other and include the true parameter values inside $1 \sigma$ confidence level contour. Adding unbound particles to the analysis shifts $M_{\mathrm{v}}$ towards higher masses (which is due to the overestimated velocity dispersion) and forces the concentration parameter to lower values (which is due to the rising dispersion profile).

\section{Direct methods of interloper removal}

\subsection{Overview}

In this section we study methods which allow us to remove a significant fraction of interlopers using some criteria. First we consider restrictions on the positions of halo particles on the velocity diagram. Given the maximum velocity available for halo particles (dynamical approach) or a distribution of halo particles on the velocity diagram (statistical approach) we impose boundaries on the area of the velocity diagram likely occupied by halo particles. Interlopers are then identified as particles from the outside of this area. Then we consider a criterion based on the way interlopers affect different mass estimators. In all these approaches the procedure of interloper removal is iterative. In each step new boundaries of the area occupied by halo particles or mass estimators are determined from the data partially cleaned of interlopers in the previous steps and the next group of interlopers is removed. All methods are supposed to converge after a few iterations when no more interlopers are identified.

Knowing which particles on the velocity diagrams are real interlopers (belonging to any of the samples defined in the previous section) we are able to study the efficiency of different methods aimed at eliminating interlopers from velocity diagrams by comparing lists of interlopers found by these methods with those identified in 3D analysis. To quantify these results we introduce three parameters: a fraction of identified interlopers $f_{i}$, a fraction of halo particles (galaxies) which were taken for interlopers by mistake $f_{\mathrm{g}}$ and a fraction of non-identified interlopers remaining in the final sample of halo members $f_{\mathrm{h}}$. For an ideal method of interloper removal we would have all interlopers identified correctly, i.e. $f_{\mathrm{i}}=1, f_{\mathrm{g}}=0$ and $f_{\mathrm{h}}=0$. In order to judge the performance of different schemes of interloper removal we calculate the mean values of the parameters and their dispersions averaging over the whole set of velocity diagrams. It should be kept in mind, however, that the values of these parameters will depend on the initial velocity cut-off used to select the data (allowing wider velocity range we would obtain higher values of $f_{\mathrm{i}}$ ). The important point is that the relative efficiency of different methods of interloper removal should not depend on this velocity cut-off. We address this issue further in the last section.

\subsection{Dynamical approach}

In this approach, we identify an interloper as a particle at a given projected radius $R$ whose velocity exceeds a maximum velocity available for halo particles at this radius. The main problem of 
this method lies in the choice of proper maximum velocity profiles. Let us consider two characteristic velocities: the circular velocity $v_{\text {cir }}$ and the infall velocity $v_{\text {inf }}$ given respectively by

$v_{\mathrm{cir}}=\sqrt{G M(r) / r}$

$v_{\text {inf }}=\sqrt{2} v_{\text {cir }}$.

Another quantity of interest would be the escape velocity $v_{\text {esc }}=$ $\sqrt{2|\Phi(r)|}$, however, as discussed by Prada et al. (2003) it does not lead to any useful criterion for interloper removal. The interpretation of the infall velocity is as follows. Assuming circular orbits of a given set of particles one can obviously recover the relation between potential and kinetic energy ( $U$ and $T$ respectively) postulated by the virial theorem: $2 T_{\text {cir }}=-U_{\text {cir. }}$. The infall velocity $v_{\text {inf }}$ is simply an upper limit to the particles' velocities for which the virial theorem equation is violated, $T_{\mathrm{inf}}=-U$ (den Hartog \& Katgert 1996; Beers et al. 1982). This limit originates from the requirement that a given particle is bound to the halo $(U+T<0)$. Note that this condition provides a stronger restriction on the maximum velocity than the general formula for the escape velocity since $v_{\text {cir }}<\sqrt{|\Phi(r)|}$. The equality $v_{\text {esc }}=v_{\text {inf }}$ would only occur if the density distribution dropped to zero at $r$ since then we would have $|\Phi(r)|=G M(r) / r$. The velocity $v_{\text {inf }}$ can therefore be viewed as an escape velocity from the mass interior to $r$.

Following den Hartog \& Katgert (1996) we introduce two formulae for the maximum velocity profile. First, assuming that the direction of particle velocity in the limit determined by $v_{\text {inf }}$ has any orientation, the maximum velocity at a given projected radius $R$ is given by

$v_{\max }=\max _{R}\left\{v_{\text {inf }}\right\}$,

where $\max _{R}$ is a maximum along the line of sight at the distance $R$ from the halo centre. A second, more restrictive criterion which gives more accurate limits at high $R \sim r_{\mathrm{v}}$ can be obtained from

$v_{\max }=\max _{R}\left\{v_{\text {inf }} \cos \theta, v_{\text {cir }} \sin \theta\right\}$,

where $\theta$ is the angle between position vector of the particle with respect to the halo centre and the line of sight. With this formula, we assume a special kinematic model which allows particles to fall onto the halo centre with velocity $v_{\text {inf }}$ or to move in a tangential direction with circular velocity $v_{\text {cir }}$.

To complete the above prescription for the maximum velocity profiles one needs to specify the mass profile. As proposed by den Hartog \& Katgert (1996), we use the mass estimator $M_{\mathrm{VT}}$ derived from the virial theorem (Limber \& Mathews 1960; Bahcall \& Tremaine 1981; Heisler et al. 1985)

$M_{\mathrm{VT}}\left(r=R_{\max }\right)=\frac{3 \pi N}{2 G} \frac{\sum_{i}\left(v_{i}-\bar{v}\right)^{2}}{\sum_{i<j} 1 / R_{i, j}}$,

where $N$ is a number of galaxies enclosed on the sky by a circle with radius $R_{\max }, v_{i}$ is the velocity of the $i$ th galaxy and $R_{i, j}$ is a projected distance between $i$ th and $j$ th galaxy. This formula is valid for spherical systems with arbitrary anisotropy. The mass profile can be simply obtained as $M(r) \approx M_{\mathrm{VT}}\left(R_{\mathrm{i}}<r<R_{i+1}\right)$, where $R_{\mathrm{i}}$ is the sequence of projected radii of particles (galaxies) in the increasing order. The virial theorem applies to a whole system and otherwise one needs to add a surface term (e.g. The \& White 1986). Recently Biviano et al. (2006) estimated its value for simulated clusters within an aperture of $1.5 \mathrm{~h}^{-1} \mathrm{Mpc}$. However, since our purpose is not to estimate accurately the

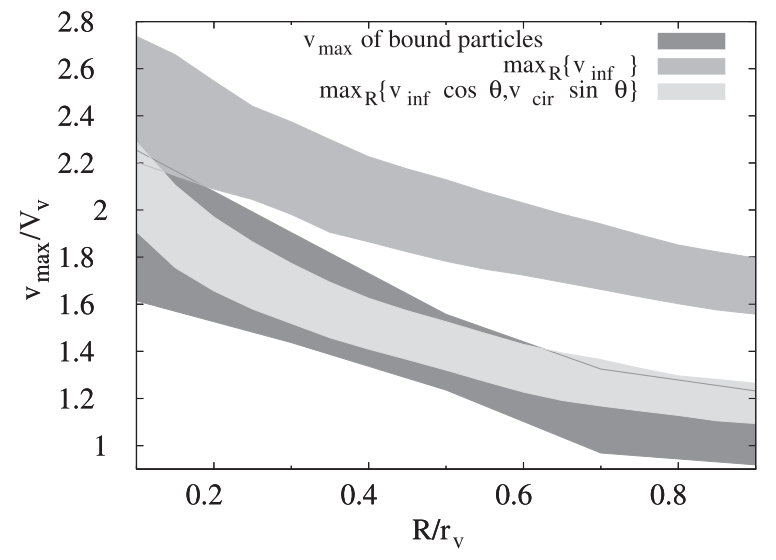

Fig. 3. Maximum velocity profiles. The dark gray area in the background indicates maximum velocity reached by bound particles. Medium and light gray strips correspond respectively to the formula (4) and (5) with the mass profile given by the mass estimator (6). Widths of the shaded areas are given by the dispersions following from averaging over the whole sample of velocity diagrams.

mass but to design a procedure for interloper removal, the formula (6) is sufficient.

Using subsamples of bound particles gathered on all mock velocity diagrams we calculate the maximum velocity profiles given by (4) and (5) with the mass profile determined by (6). The results, expressed in units of circular velocity at the virial radius $V_{\mathrm{v}}$, are shown in Fig. 3 as medium and light gray strips respectively, whereas the dark gray profile seen in the background of this plot indicates the average maximum velocity reached by bound particles (including bound particles beyond $r_{\mathrm{v}}$ ) on any velocity diagram. Widths of all three areas are given by the dispersions resulting from averaging the maximum velocity profiles over the whole sample of velocity diagrams. It is clear that formula (5) is expected to work best in removing interlopers since its profile (light gray) coincides almost exactly with the maximum velocity reached by bound particles (dark gray). On the other hand, the profile generated by formula (4) seems too conservative to be useful.

Figure 4 illustrates successive steps of interloper removal with the maximum velocity (5) (hereafter $v_{\max }(1)$ method) for one of mock velocity diagrams with rather large number of unbound particles. The top and bottom panels show mass profiles and maximum velocity profiles separating interlopers from halo particles on the velocity diagram for successive iterations of this method marked with numbers. The final virial mass given simply by the value of estimator $M_{\mathrm{VT}}$ for $R_{\max }=r_{\mathrm{v}}$ is equal to $8.35 \times 10^{14} M_{\odot}$ which is a few times lower than for the total contaminated sample (first iteration in Fig. 4) and reasonably close to the real value of the virial mass found in $3 \mathrm{D}$ analysis, $5.35 \times 10^{14} M_{\odot}$. Note that the mass estimator $M_{\mathrm{VT}}$ is known to overestimate the true mass due to the neglect of the surface term (see Biviano et al. 2006) and a more reliable final estimate can in general be obtained by fitting velocity moments (Sanchis et al. 2004; Łokas et al. 2006).

With this method, on average 73 percent of unbound particles are identified and removed from a sample and only around 1 percent of bound particles are taken for interlopers and lost from the velocity diagram so that the final samples include only around 2-3 percent of unbound particles (see Table 1 for details). Note that the fraction of removed interlopers $f_{\mathrm{i}}$ is limited in principle to values lower than about 75 percent because roughly $1 / 4$ of unbound particles within the observation cylinder with 

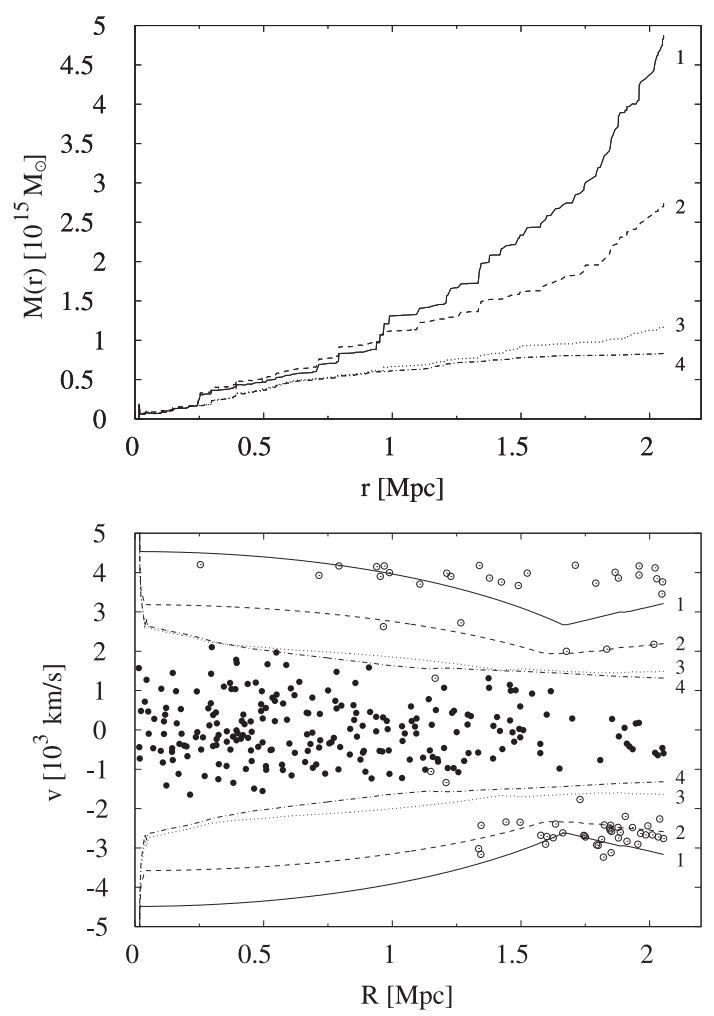

Fig. 4. Illustration of successive steps in $v_{\max }(1)$ method of interloper removal for halo 6 in projection along the $y$ axis. The method uses the maximum velocity profile (5) and mass profile (6). The top and bottom panels show successive mass profiles and maximum velocity profiles separating interlopers from halo particles respectively. Filled and empty circles mark particles bound and unbound to the halo. Numbers indicate successive steps of the procedure which are described by the lines of different types. Final maximum velocity profiles (number 4) are drawn with dashed-dotted lines.

velocity cut-off $4000 \mathrm{~km} \mathrm{~s}^{-1}$ are within the envelope of bound velocities and therefore inaccessible for direct methods of interloper identification. Since $f_{\mathrm{i}}=73$ percent available in this approach is very close to the expected maximum, the method presented above is possibly the most effective. As expected from Fig. 3, the method of interloper removal based on profile (4) is too conservative and on average identifies much less interlopers than the previous one (see $v_{\max }(2)$ method in Table 1 ).

\subsection{Statistical approach}

The idea of this approach is to use the information about the distribution of halo particles on the velocity diagram to distinguish between the probable halo particles and interlopers. The first scheme along these lines was introduced by Yahil \& Vidal (1977) who proposed to identify interlopers as galaxies with velocities from the outside of the range $\pm 3 \sigma_{\text {los }}$ around the mean cluster velocity, where $\sigma_{\text {los }}$ is the projected velocity dispersion of galaxies in the cluster given by the standard unbiased estimator. In this formulation the method is model-independent so that the data are self-verified as far as the interloper removal is concerned.

It is easy to generalize the above prescription to the case of data gathered in $n$ radial bins so that $3 \sigma_{\text {los }}$ procedure could be applied in each bin independently in the way proposed by Yahil $\&$ Vidal. This modification allows us to take into account dependence of the velocity dispersion on $R$. However, increasing the
Table 1. Results of different procedures of interloper removal in terms of the fraction of removed interlopers $f_{\mathrm{i}}$, the fraction of halo particles incorrectly identified as interlopers $f_{\mathrm{g}}$ and the fraction of non-identified interlopers remaining in the final sample of halo members $f_{\mathrm{h}}$. The table lists both the mean values $\langle f\rangle$ and the dispersions $\sigma_{\mathrm{f}}$ of the parameters obtained in the analysis of 30 mock velocity diagrams. For all seven methods of interloper removal considered in Sect. 3 results are quantified for three different definitions of interlopers, particles beyond $r_{\mathrm{v}}$ or $2 r_{\mathrm{v}}$ and unbound particles, as marked in the second column (interloper type $-\mathrm{i} / \mathrm{t}$ ) by $r_{\mathrm{v}}, 2 r_{\mathrm{v}}$ and $v_{\text {esc }}$ respectively.

\begin{tabular}{llllllrl}
\hline \hline Method & $\mathrm{i} / \mathrm{t}$ & $\left\langle f_{\mathrm{i}}\right\rangle$ & $\sigma_{f_{\mathrm{i}}}$ & $\left\langle f_{\mathrm{g}}\right\rangle$ & $\sigma_{f_{\mathrm{g}}}$ & $\left\langle f_{\mathrm{h}}\right\rangle$ & $\sigma_{f_{\mathrm{h}}}$ \\
\hline$v_{\max }(1)$ & $r_{\mathrm{v}}$ & 23 & 17 & 1.1 & 1.5 & 19.7 & 8.7 \\
& $2 r_{\mathrm{v}}$ & 48 & 26 & 1.1 & 1.4 & 7.7 & 7.8 \\
& $v_{\text {esc }}$ & 73 & 23 & 1.0 & 1.4 & 2.4 & 4.0 \\
$v_{\max }(2)$ & $r_{\mathrm{v}}$ & 13 & 11 & 0.0 & 0.0 & 21.9 & 9.4 \\
& $2 r_{\mathrm{v}}$ & 30 & 22 & 0.0 & 0.0 & 10.2 & 9.0 \\
& $v_{\text {esc }}$ & 48 & 29 & 0.0 & 0.0 & 5.2 & 6.5 \\
$3 \sigma_{\text {los }}(5)$ & $r_{\mathrm{v}}$ & 17 & 9 & 0.9 & 1.4 & 21.3 & 8.9 \\
& $2 r_{\mathrm{v}}$ & 37 & 21 & 0.8 & 1.2 & 9.4 & 8.3 \\
& $v_{\text {esc }}$ & 58 & 27 & 0.7 & 1.0 & 4.3 & 5.3 \\
$3 \sigma_{\text {los }}(10)$ & $r_{\mathrm{v}}$ & 19 & 10 & 1.6 & 2.4 & 21.0 & 8.8 \\
& $2 r_{\mathrm{v}}$ & 40 & 22 & 1.5 & 2.4 & 9.1 & 8.3 \\
& $v_{\text {esc }}$ & 63 & 27 & 1.4 & 2.2 & 3.9 & 5.1 \\
$3 \sigma_{\text {los }}(R)$ & $r_{\mathrm{v}}$ & 19 & 17 & 0.2 & 0.5 & 20.4 & 9.2 \\
& $2 r_{\mathrm{v}}$ & 40 & 25 & 0.2 & 0.4 & 8.6 & 8.4 \\
& $v_{\text {esc }}$ & 61 & 28 & 0.2 & 0.3 & 3.4 & 4.4 \\
$v_{\text {lim }}$ & $r_{\mathrm{v}}$ & 19 & 17 & 0.3 & 0.5 & 20.4 & 9.2 \\
& $2 r_{\mathrm{v}}$ & 41 & 25 & 0.3 & 0.5 & 8.5 & 8.3 \\
& $v_{\text {esc }}$ & 62 & 27 & 0.3 & 0.4 & 3.4 & 4.4 \\
$M_{\mathrm{P}} / M_{\mathrm{VT}}$ & $r_{\mathrm{v}}$ & 18 & 7 & 1.2 & 1.2 & 21.1 & 8.9 \\
& $2 r_{\mathrm{v}}$ & 40 & 22 & 1.2 & 1.3 & 9.3 & 8.3 \\
& $v_{\text {esc }}$ & 65 & 26 & 1.2 & 1.2 & 4.1 & 5.8 \\
\hline
\end{tabular}

number of bins we let the dispersion in the outer part of velocity diagram be much more overestimated by interlopers. A way to overcome this problem is to use subsequently different numbers of bins. The dispersion in wide bins (when there is a small number of bins) is less biased by interlopers so in this case we remove interlopers efficiently. On the other hand, using narrow bins (when there is a larger number of bins) we measure the dispersion locally taking into account the dependence of $\sigma_{\text {los }}$ on $R$.

In each step of this method we use the following estimators of mean velocity and velocity dispersion

$$
\begin{aligned}
\bar{v}_{i} & =\frac{\sum_{j=1}^{j=m-1} v_{i, j}}{m-1} \\
\sigma_{\mathrm{los}, i}^{2} & =\frac{\sum_{j=1}^{j=m-1}\left(v_{i, j}-\bar{v}_{i}\right)^{2}}{m-2},
\end{aligned}
$$

where $i$ is a number of bin, $m$ is a number of datapoints per bin and $v_{i, j}$ is the sequence of velocities in the $i$ th bin with the most outlying from the mean value in the last position so that following the prescription of Yahil \& Vidal we do not take into account these velocities in estimating the dispersion. For each number of bins changing in a given step from $n_{\min }$ to $n_{\max }$ we remove particles with velocities from the outside of the range $\pm 3 \sigma_{\text {los }, i}$ around $\bar{v}_{i}:\left|v_{i, j}-\bar{v}_{i}\right|>3 \sigma_{\text {los }, i}$. The procedure converges after a few steps when no more interlopers are found in any bin.

We find that in order to remove even strongly clustered groups of interlopers (like the ones on the velocity diagram of halo 6 in projection along $y$ axis seen in the middle left panel of Fig. 6) it is necessary to fix $n_{\min }=1$ which corresponds to the 

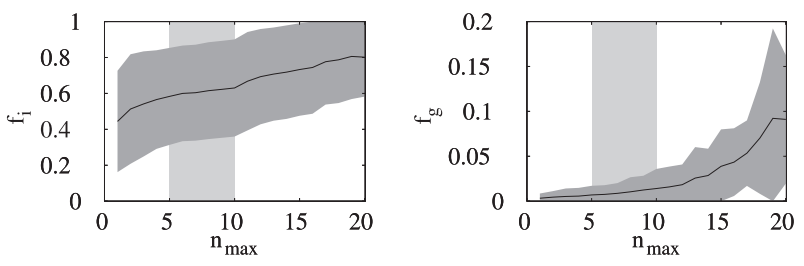

Fig. 5. Dependence of the results of the interloper removal method based on the analysis of the velocity dispersion of binned data on the maximum number of bins $n_{\max }$. The left panel shows the fraction of identified unbound particles $f_{\mathrm{i}}$ and the right one the fraction of bound particles taken for interlopers by mistake $f_{\mathrm{g}}$. Widths of both profiles (dark gray) are given by the dispersions following from averaging over the sample of velocity diagrams. Light gray strip in the background indicates the best range of $n_{\max }$.

original approach of Yahil \& Vidal (1977). To fix a maximum number of bins $n_{\max }$ we consider the dependence of $f_{\mathrm{i}}$ and $f_{\mathrm{g}}$ on different choices of the value of $n_{\max }$. The results are shown in Fig. 5 in the form of dark gray profiles. Using them we can easily find the values of $n_{\max }$ for which the procedure gives possibly high $f_{\mathrm{i}}$ and low $f_{\mathrm{g}}$. This range of $n_{\max }$ is marked with a light gray strip in the background of the plot. Applying its lower limit $\left(n_{\max }=5\right)$, for which $f_{\mathrm{i}}$ profile begins inclining towards $n_{\max }$ axis, leads to slightly conservative method of interloper removal with average rate of unbound particle identification $f_{\mathrm{i}}=58$ percent (see $3 \sigma_{\text {los }}(5)$ method in Table 1). In the upper limit of this range $\left(n_{\max }=10\right)$, when $f_{\mathrm{g}}$ starts increasing rapidly, algorithm is a bit more restrictive and allows to remove on average 63 percent of unbound particles with the rate of misidentification $f_{\mathrm{g}}$ comparable to the result of $v_{\max }(1)$ method (see $3 \sigma_{\text {los }}(10)$ method in Table 1 for more details).

Recently Łokas et al. (2006) generalized the $\pm 3 \sigma_{\text {los }}$ rule of interloper identification to a continuous velocity dispersion profile: $\pm 3 \sigma_{\text {los }}(R)$, where $\sigma_{\text {los }}(R)$ is the projected isotropic solution to Jeans equation parametrized by $M_{\mathrm{v}}$ and $c$ (Binney \& Mamon 1982; see also Prugniel \& Simien 1997; Łokas \& Mamon 2001; Mamon \& Łokas 2005) fitted to the binned data. Assumption of isotropic orbits allows us to break the degeneracy between $c$ and the anisotropy and to trace accurately the shape of the velocity dispersion profile with the $c$ parameter only. We find that in some cases of velocity diagrams with strong interloper contamination this procedure stops too early because of the overestimation of the velocity dispersion profile. To fix this problem we propose to fit $\sigma_{\text {los }}(R)$ to an incomplete dispersion profile after rejecting a few outer data points which are most contaminated. In our case we proceeded with the fitting for at least 6 data points to the maximum of 10 (always with 30 particles per bin) and then used the mean values of $M_{\mathrm{v}}$ and $c$ obtained for $k=6-10$ data points. The mean values were weighted with the goodness of fit measure $\chi_{\min }^{2} /(k-2)$ so that parameter values coming from worse-quality fits caused mainly by the presence of interlopers were naturally attenuated.

Figure 6 illustrates this approach both in the form of the final $\pm 3 \sigma_{\text {los }}(R)$ lines on the velocity diagram (left column) and the velocity dispersion profiles obtained in subsequent steps of this procedure (right column) for halo 6 in three projections. The procedure allows to remove on average 61 percent of unbound particles from a given velocity diagram with the rate of misidentification of only around 0.2 percent ( $\operatorname{see} 3 \sigma_{\text {los }}(R)$ method in Table 1 ). Note that $f_{\mathrm{i}}$ in this case achieves values similar to those obtained with the $3 \sigma_{\text {los }}(10)$ method, while $f_{\mathrm{g}}$ is much smaller.
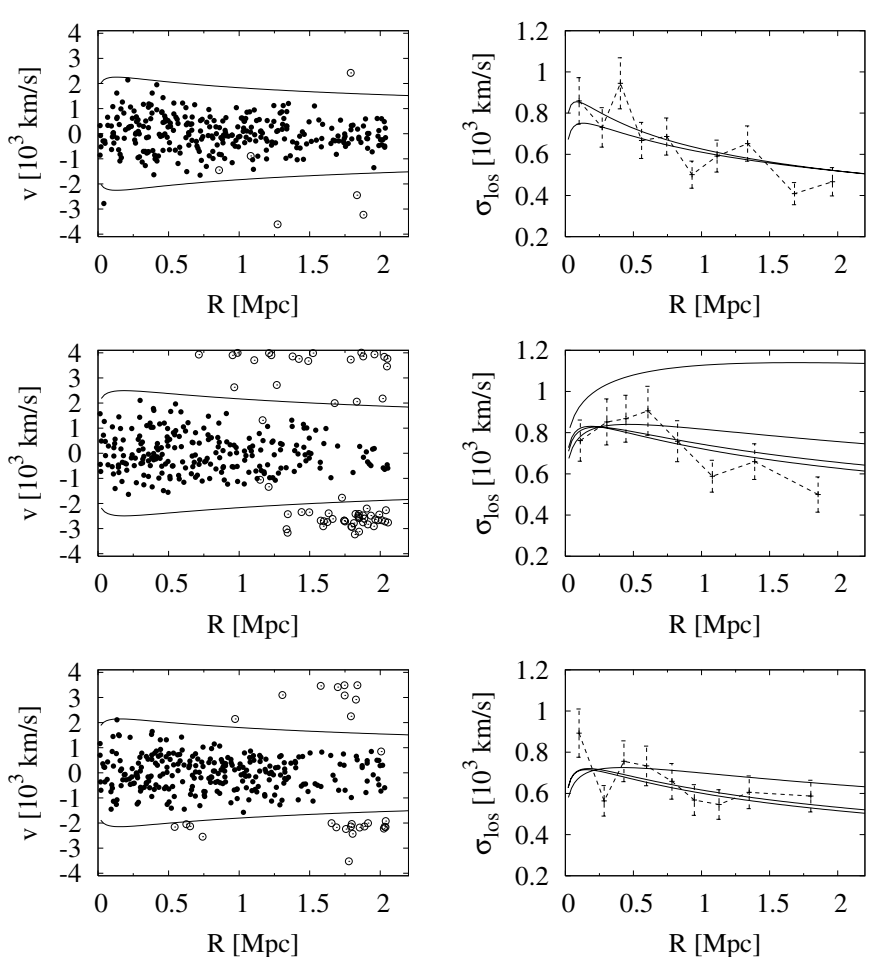

Fig. 6. Results of the $3 \sigma_{\text {los }}(R)$ method for halo 6 in three projections ( $x, y$ and $z$ from top to bottom). Left column panels show velocity diagrams with final $\pm 3 \sigma_{\text {los }}(R)$ lines separating interlopers from halo particles. Filled and empty circles on velocity diagrams indicate bound and unbound particles respectively. Right column panels show the line-ofsight velocity dispersion profiles obtained in successive steps of the procedure (solid lines from top to bottom). The dashed line with error bars is the dispersion profile measured for bound particles.

\subsection{Generalized statistical approach}

The methods presented in the above subsection assume implicitly that the projected distribution of halo particles does not depend on the projected radius. In the following, we reformulate this approach properly, taking into account the full dependence of the distribution of halo particles on $R$ and $v$. A natural extension of the criterion introduced by Yahil \& Vidal (1977) is then given by the boundary line $\pm v_{\text {lim }}(R)$ which determines an area occupied by halo particles on the velocity diagram with some probability $p_{\lim }(R)$. Conditions for $v_{\lim }(R)$ can be written as follows:

$$
\begin{aligned}
\int_{0}^{R_{\max }} \int_{-v_{\lim }(R)}^{v_{\lim }(R)} p_{R, v} \mathrm{~d} v \mathrm{~d} R & =p_{\lim } \\
p_{R, v}\left[R, v_{\lim }(R)\right] \mathrm{d} v \mathrm{~d} R & =C \mathrm{~d} v \mathrm{~d} R
\end{aligned}
$$

where $p_{R, v}$ is the projected probability distribution of halo particles and $C$ is its constant value along the boundary line $\pm v_{\text {lim }}$. Second equation in (9) is necessary to fully constrain the final solution.

In order to find a useful analytical approximation for $p_{R, v}$ we first assume that the probability of finding a halo particle inside an infinitesimal range of radius $[R, R+\mathrm{d} R]$ of a cylinder of observation with radius $R_{\max }$ is given by

$p_{R} \mathrm{~d} R=2 \pi R \frac{\Sigma_{M}(R, c)}{M_{\mathrm{P}}\left(R_{\max }, c\right)} \mathrm{d} R$,

where $\Sigma_{M}(R, c)$ and $M_{\mathrm{P}}\left(R_{\max }, c\right)$ are surface density and projected mass inferred from this surface density; in our case they 


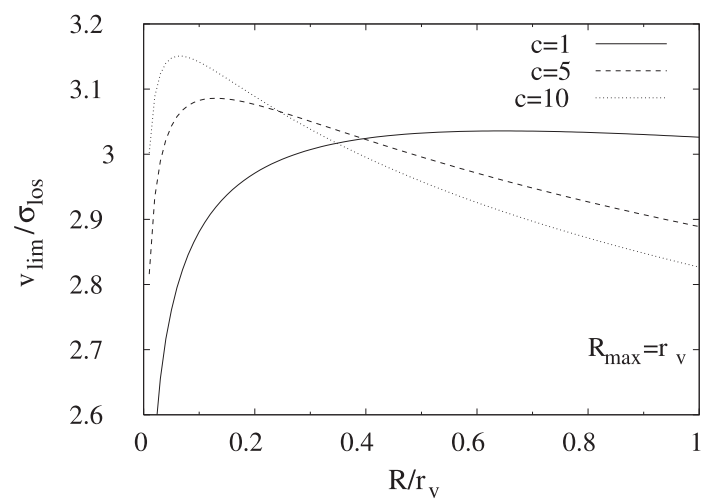

Fig. 7. Boundary lines $v_{\text {lim }}$ of the area occupied by halo particles with probability $p_{\text {lim }}=0.9973$ for three values of the concentration parameter $c=1,5,10$. The velocity diagram is assumed to have a cut-off in radius at $R_{\max }=r_{\mathrm{v}}$ and $v_{\mathrm{lim}}$ is expressed in units of the local value of the projected velocity dispersion $\sigma_{\text {los }}(R)$.

follow from the NFW density formula (see Bartelmann 1996; Łokas \& Mamon 2001). To be precise, Eq. (10) is satisfied best by dark matter particles and could be less suitable for description of spatial distribution of galaxies. However, cluster data are consistent with galaxy distribution being given by the NFW profile and mass-to-number density being constant within the virial radius (see e.g. Biviano \& Girardi 2003) so that formula (10) seems to be useful also in the context of real data.

Second, we assume that the distribution of the line-of-sight velocity at a given radius $R$ (i.e. the conditional probability of a particle having a velocity $v$ if it is at projected distance $R$ ) can be well approximated by a Gaussian distribution

$p_{v} \mathrm{~d} v=\frac{1}{\sqrt{2 \pi} \sigma_{\text {los }}(R, c)} \exp \left(-\frac{v^{2}}{2 \sigma_{\text {los }}^{2}(R, c)}\right) \mathrm{d} v$,

where $\sigma_{\text {los }}(R)$ is the projected isotropic solution to the Jeans equation. Although this approximation is not exactly valid since departures from Gaussianity are seen both in simulated haloes and real clusters (Kazantzidis et al. 2004; Wojtak et al. 2005; Hansen et al. 2006; Łokas et al. 2006) we claim it is sufficient for our needs. In particular, one can show that for isotropic orbits the projected velocity distribution following from the distribution function for the NFW profile (see Łokas \& Mamon 2001; Widrow 2000) is remarkably close to a Gaussian in a wide range of radii.

Finally, combining the formulae for $p_{R}$ and $p_{v}$ we get a heuristic expression for the projected probability distribution:

$p_{R, v} \mathrm{~d} R \mathrm{~d} v=p_{R} p_{v} \mathrm{~d} R \mathrm{~d} v$.

One can immediately check that the normalization condition on the available area of the velocity diagram is satisfied automatically with sufficiently high numerical precision since $v_{\max }(R) \gtrsim$ $4 \sigma_{\text {los }}(R)$ :

$\int_{0}^{R_{\max }} \int_{-v_{\max }(R)}^{v_{\max }(R)} p_{R, v} \mathrm{~d} v \mathrm{~d} R=1$.

Note that $p_{R, v}$ given by (12), by analogy with results obtained by Maoz \& Bekenstein (1990), maximizes Shannon's entropy for known functions $\Sigma_{M}(R)$ and $\sigma_{\text {los }}(R)$. In the language of the information theory this means that $p_{R, v}$ is the most plausible probability distribution given $\Sigma_{M}(R)$ and $\sigma_{\text {los }}(R)$.

Substituting (12) into (9) we derive $v_{\lim }(R) / \sigma_{\text {los }}(R)$ from the second of these equations. Introducing this expression to
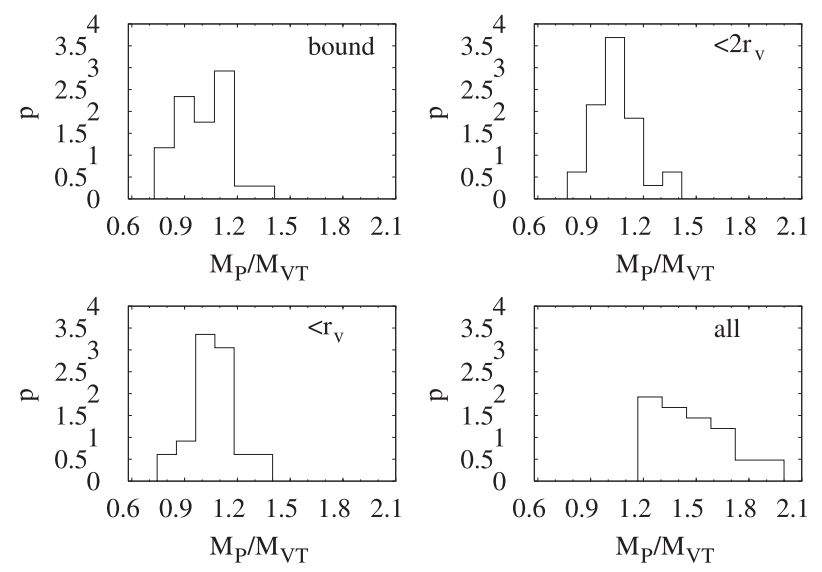

Fig. 8. Histograms of the ratio $M_{\mathrm{P}} / M_{\mathrm{VT}}$ for different subsamples of particles. Subsamples are identified in the upper right corner of each panel: the lower right panel is for all particles, upper left for bound particles and the remaining two for particles inside $r_{\mathrm{v}}$ or $2 r_{\mathrm{v}}$. All histograms are normalized to unity.

the first equation we calculate numerically the constant $C$ and in the end evaluate the whole profile $v_{\text {lim }}(R) / \sigma_{\text {los }}(R)$. Figure 7 shows numerical solutions assuming cut-off radius $R_{\max }=r_{\mathrm{v}}$ and $p_{\lim }=0.9973$, a value corresponding to the $\pm 3 \sigma$ range for a Gaussian distribution. We see that in general $v_{\lim }(R) / \sigma_{\operatorname{los}}(R)$ departs significantly from the value of 3 . Only in the limit of $p_{R} \sim$ const. we recover the continuous version of the criterion introduced by Yahil \& Vidal (1977): $v_{\text {lim }}(R)=3 \sigma_{\text {los }}(R)$. We find that using the exact solution to the set of Eq. (9) does not improve the performance of the method, the numbers of interlopers removed in this case are similar as for the simpler $3 \sigma_{\text {los }}$ schemes (see $v_{\text {lim }}$ method in Table 1).

\subsection{Mass estimators as indicators of interloper fraction}

In this subsection we study the effect of interlopers on the values of two standard mass estimators and use the results to construct another method of interloper removal. The estimators we use are the virial mass $M_{\mathrm{VT}}$ expressed by formula (6) and the projected mass $M_{\mathrm{P}}$ for isotropic orbits given by (Heisler et al. 1985; Perea et al. 1990)

$M_{\mathrm{P}}=\frac{32}{\pi G N} \Sigma_{i}\left(v_{i}-\bar{v}\right)^{2} R_{i}$

where $v_{i}$ and $R_{i}$ are the velocity and projected radius of the $i$ th galaxy, $N$ is the number of galaxies in the sample.

Both mass estimators are sensitive to the presence of interlopers, but each of them in a different way (Perea et al. 1990). $M_{\mathrm{P}}$ is considerably more overestimated than $M_{\mathrm{VT}}$ so that interlopers effectively give rise to the increase of $M_{\mathrm{P}} / M_{\mathrm{VT}}$ ratio above 1 . In principle, it is impossible to relate the value of this ratio to the number of any kind of interlopers because of strong degeneracy: a given value of $M_{\mathrm{P}} / M_{\mathrm{VT}}$ can be reproduced with various numbers of interlopers and their different distributions on the velocity diagram. Nevertheless, it is interesting to study the distributions of the ratio $M_{\mathrm{P}} / M_{\mathrm{VT}}$ for four different particle subsamples: all particles, particles inside $2 r_{\mathrm{v}}$ or $r_{\mathrm{v}}$ and bound particles.

The results are shown in Fig. 8 in the form of histograms (normalized to unity) constructed from the data in our 30 velocity diagrams. As we can see, there is no significant difference between histograms obtained for all three subsamples with different types of interlopers subtracted. All of them have a maximum 

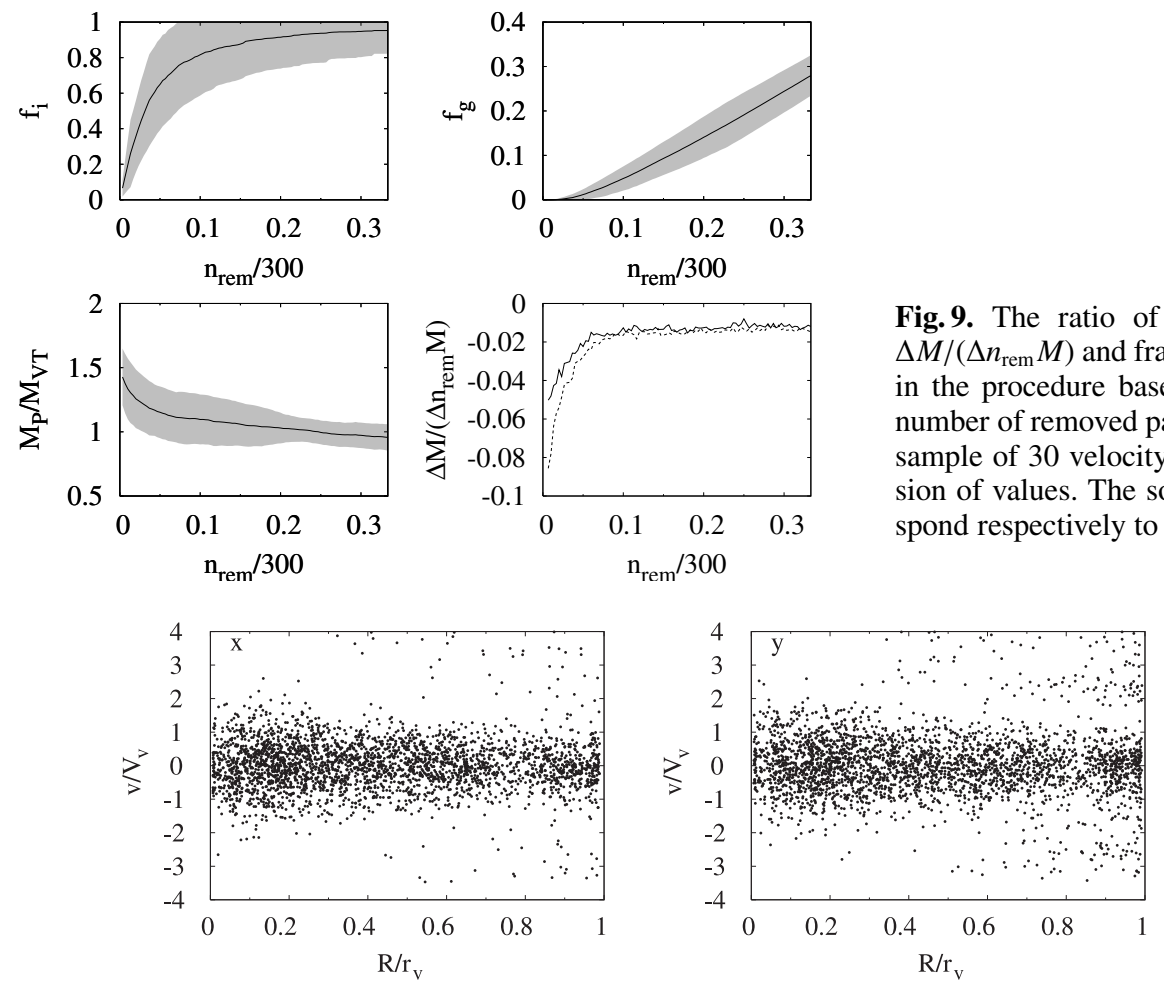

Fig. 9. The ratio of mass estimators $M_{\mathrm{P}}$ and $M_{\mathrm{VT}}$, their derivatives $\Delta M /\left(\Delta n_{\mathrm{rem}} M\right)$ and fractions of unbound $f_{\mathrm{i}}$ and bound $f_{\mathrm{g}}$ particles removed in the procedure based on jackknife statistics as a function of the total number of removed particles $n_{\text {rem }}$. All profiles are averaged over the whole sample of 30 velocity diagrams and the shaded areas indicate the dispersion of values. The solid and dashed lines in the lower right panel correspond respectively to $M_{\mathrm{VT}}$ and $M_{\mathrm{P}}$.

Fig. 10. Stacked velocity diagrams in projection along $x, y$ and $z$ axes.

at around $M_{\mathrm{P}} / M_{\mathrm{VT}}=1$ and a spread between $\sim 0.8$ and $\sim 1.4$. Including unbound particles in the analysis, we get a highly asymmetric histogram, shifted to the range $1.2-2.0$ with a maximum at $\sim 1.2$. These results prove that the unbound particles are the ones that give rise to the overestimation of the ratio of mass estimators and therefore the ratio $M_{\mathrm{P}} / M_{\mathrm{VT}}$ can be a useful indicator of the contamination of a sample with unbound particles.

This phenomenological relation between the ratio of mass estimators and the presence of unbound particles in the sample can be used to construct a procedure which eliminates this kind of interlopers from the velocity diagram. The prescription for such interloper identification was proposed by Perea et al. (1990) and is based on jackknife statistics. Let $\left\{R_{i}, v_{i}\right\}$ be a sequence of data, where $i$ goes from 1 to $n$. Following the jackknife technique, we calculate $n$ values of both mass estimators which correspond to $n$ subsequences with one data point excluded. Finally we identify as an interloper the particle for which the corresponding subsequence is the source of the most discrepant value of one of the estimators with respect to the mean value. In the next step, the same procedure is applied to a new data set with $n-1$ particles.

The main problem of this procedure lies in defining properly the condition for stopping the algorithm. In order to specify it we calculate the ratio $M_{\mathrm{P}} / M_{\mathrm{VT}}$ and the fractions $f_{\mathrm{i}}$ and $f_{\mathrm{g}}$ (for interlopers defined as unbound particles) as functions of the number of removed particles $n_{\text {rem }}$ determined by the jackknife technique. Figure 9 shows the results averaged over all 30 velocity diagrams so that the shaded areas indicate the dispersion of the values. From the behaviour of all profiles we infer that the most accurate moment of convergence (around $n_{\text {rem }} / 300=0.05$ ) coincides clearly with a characteristic knee-like point of $M_{\mathrm{P}} / M_{\mathrm{VT}}$ and $\Delta M / M$ profiles. This point is usually more recognizable in the case of single velocity diagrams so that it can be effectively used to judge where the algorithm should be stopped (see
Wojtak \& Łokas 2006). Taking $n_{\text {rem }} / 300=0.05$ we are able to remove on average 65 percent of unbound particles with around 1 percent of bound particles taken for interlopers by mistake (see $M_{\mathrm{P}} / M_{\mathrm{VT}}$ method in Table 1).

\section{Indirect methods of interloper treatment}

\subsection{The distribution of interlopers}

The key idea of the indirect approach is to treat interlopers statistically in the proper dynamical analysis so that no particles need to be rejected (van der Marel et al. 2000; Prada et al. 2003). In all versions of this method one assumes that the probability distribution $p(R, v)$ of particles seen on the velocity diagram consists of two terms which describe the distribution of halo particles and the distribution of interlopers.

Such an analysis requires numerous and regular samples of both kinds of particles on the velocity diagram and cannot be done for a single object where the distribution of interlopers can be highly irregular. We therefore stacked all 30 velocity diagrams into three composite ones in each projection separately. All radii and velocities were rescaled by $r_{\mathrm{v}}$ and $V_{\mathrm{v}}$ respectively so that the mass dependence is factored out. This procedure is commonly applied to the cluster data to improve the statistics and to study the typical properties of clusters which are expected to scale with mass (e.g. Mahdavi \& Geller 2004; Łokas et al. 2006). Figure 10 shows our three stacked velocity diagrams in projection along the $x, y$ and $z$ axes.

For each of these diagrams, we calculate surface density profiles and velocity histograms for all three kinds of interlopers (Fig. 11). It is clearly seen that unbound particles are the type of interlopers with the most uniform surface density and velocity distribution, whereas particles from the samples beyond $2 r_{\mathrm{v}}$ or $r_{\mathrm{v}}$ are considerably concentrated in the vicinity of the halo mean velocity (see also Cen 1997; Diaferio et al. 1999; and 

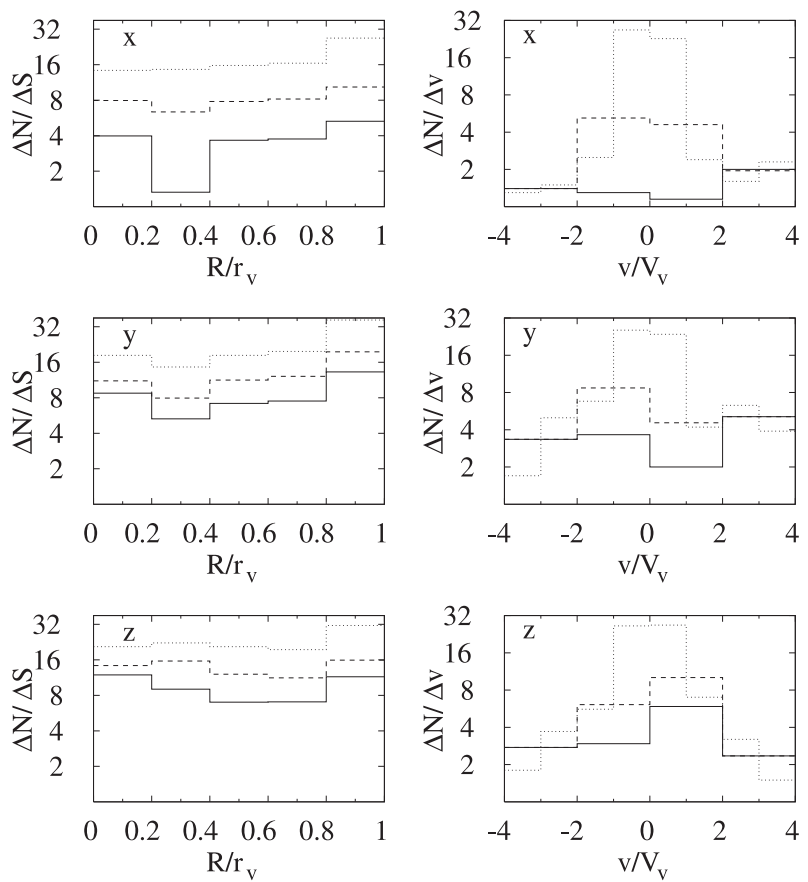

Fig. 11. Surface density (left column) and velocity histograms (right column) of interlopers on stacked velocity diagrams in different projections marked in the upper left corner of each panel. Solid, dashed and dotted lines correspond to unbound particles and particles beyond $2 r_{\mathrm{v}}$ and $r_{\mathrm{v}}$ respectively. Both surface density and velocity histograms are normalized to the number of a given type of interlopers averaged over 10 velocity diagrams in a given projection.

Chen et al. 2006). The uniformity of the distribution of interlopers on the velocity diagram is the most natural assumption and has been used previously in the construction of the probability distribution of interlopers (Mahdavi \& Geller 2004). We can see that this hypothesis agrees well with a distribution of unbound particles.

\subsection{Estimation of the velocity dispersion profile with a uniform background of interlopers}

This method was originally introduced by Prada et al. (2003) in the context of measuring the velocity dispersion profile of satellite galaxies. Following these authors we assume that the distribution of particles is given by the sum of a Gaussian part for halo particles and a constant background describing interlopers. Let $f_{R}(v) \mathrm{d} v$ be the conditional probability of finding any type of particle in the infinitesimal range $[v, v+\mathrm{d} v]$ at a given radius $R$

$f_{R}(v) \mathrm{d} v=\alpha(R) p_{v} \mathrm{~d} v+[1-\alpha(R)] \frac{\mathrm{d} v}{2 v_{\max }}$,

where $p_{v}$ is the Gaussian distribution given by (11) with dispersion equal to $\sigma_{\text {los }}(R)$ and the local mean velocity $\mu(R), v_{\max }$ is the maximum velocity available on the velocity diagram and $\alpha(R)$ has a simple interpretation of the probability of finding a halo particle at a given radius $R$. Note that in this case the normalization condition is valid only for a fixed radius $R$. All radiusdependent quantities are estimated in radial bins by fitting formula (15) to a velocity histogram in a given bin by minimizing the $\chi^{2}$ function. We use 10 radial bins with 300 data points in each of them.

An example of the velocity histogram together with the fitted probability distribution given by (15) is shown in Fig. 12. The

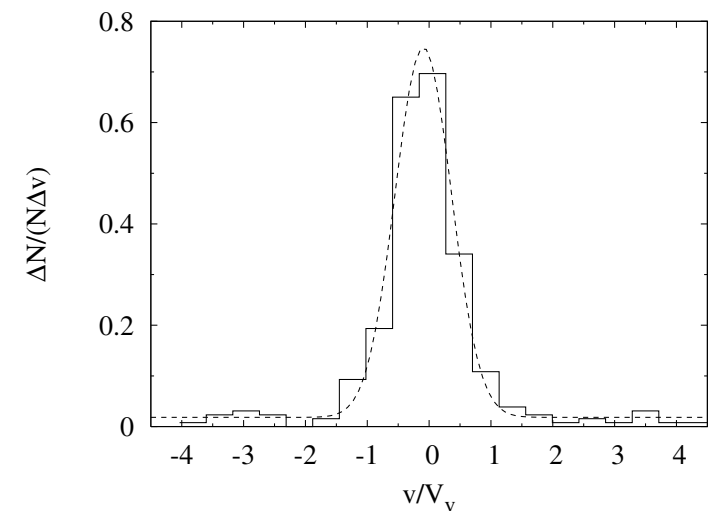

Fig. 12. The velocity histogram and the fitted probability distribution given by (15) for the outer bin of a stacked velocity diagram in projection along the $x$ axis.

dispersion profiles obtained in the fitting procedure are shown in the left column of Fig. 13 (filled circles). For comparison, we also plot velocity dispersion profiles of bound particles with shaded areas corresponding to $\pm 3 \sigma$ departures from the mean. As we can see, all profiles determined in the fitting procedure decline properly with radius and trace well the dispersion profiles of bound particles. Interestingly, some clearly overestimated values of $\sigma_{\mathrm{los}}$ and $\alpha$ probability appear in the same range of radii for the case of projection along the $z$ axis which is an effect of local irregularities in the velocity distribution of unbound particles.

It seems interesting to compare $\alpha(R)$ profiles obtained in the fitting procedure with those directly measured from the data for different types of interlopers. Using frequency definition of probability $\alpha$ is expressed as

$\alpha(R)=\frac{N_{\mathrm{g}}(R)}{N_{\mathrm{g}}(R)+N_{\mathrm{i}}(R)}$,

where $N_{\mathrm{g}}$ and $N_{\mathrm{i}}$ are numbers of halo particles and interlopers defined by a given criterion respectively. In the right column panels of Fig. 13 we plot $\alpha$ profiles estimated in radial bins with interlopers defined as: unbound particles (solid lines), particles beyond $2 r_{\mathrm{v}}$ (dashed lines) and $r_{\mathrm{v}}$ (dotted lines). Profiles obtained in the fitting procedure are indicated with broken dashed-dotted lines. We can clearly see that, as expected, the fitted $\alpha$ profiles reproduce direct measurements best for bound particles.

We therefore conclude that the favoured group of interlopers in this approach consists of unbound particles, whereas most particles from the outside of the virial region, but bound to a given halo contribute to the Gaussian part of the distribution function $f_{R}(v)$ and in fact are used in the estimation of the dispersion profile. This situation is difficult to avoid; wishing to include particles beyond $2 r_{\mathrm{v}}$ or $r_{\mathrm{v}}$ as interlopers one would have to introduce another Gaussian-like distribution of interlopers in velocity space (see velocity histograms in Fig. 11). This, however, would cause strong degeneracy since both halo particles and interlopers would be described by very similar distributions.

\subsection{Bayesian technique}

In this subsection we study the elegant approach of statistical treatment of interlopers originally proposed by van der Marel et al. (2000) and Mahdavi \& Geller (2004). This method is based on the Bayes technique which allows us to determine the probability distribution in the parameter space of a particular model given the measured data. Consider the parameter set $\boldsymbol{a}$ and data 

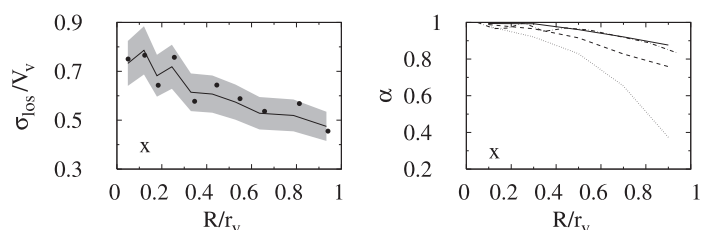

$\mathrm{R} / \mathrm{r}_{\mathrm{v}}$
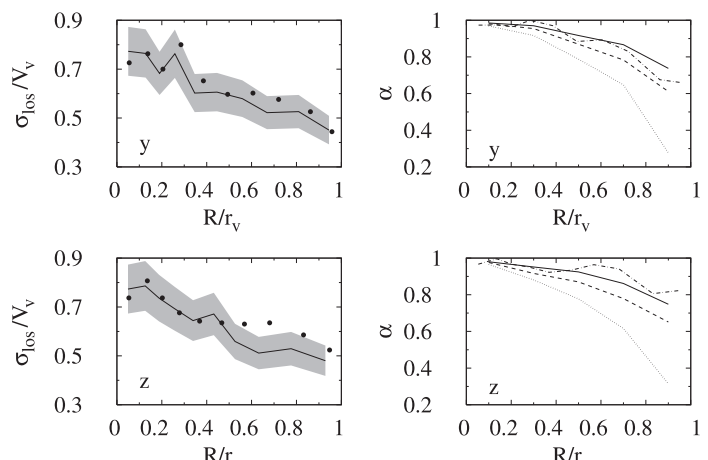

Fig. 13. Left column panels show dispersion profiles obtained by fitting the probability distribution (15) to velocity histograms for composite haloes in different radial bins (filled circles). Black lines plot dispersion profiles of bound particles with shaded strips indicating the $3 \sigma$ range of variability among the velocity diagrams. Right column panels show profiles of the probability $\alpha(R)$ measured for particles beyond $r_{\mathrm{v}}$ (dotted lines) or $2 r_{\mathrm{v}}$ (dashed lines) and for unbound particles (solid lines). Dashed-dotted broken lines represent results of the fitting procedure. Each row of panels corresponds to a different projection direction of the stacked velocity diagram marked in the bottom left corner.

set $\left\{\boldsymbol{x}_{i}\right\}$. Following Bayes theorem the probability of getting certain values of parameters $\boldsymbol{a}$ given data sequence $\left\{\boldsymbol{x}_{i}\right\}$ is

$p\left(\boldsymbol{a} \mid\left\{\boldsymbol{x}_{i}\right\}\right)=\frac{p(\boldsymbol{a})}{p\left(\left\{\boldsymbol{x}_{i}\right\}\right)} \Pi_{i} p\left(\boldsymbol{x}_{i} \mid \boldsymbol{a}\right)$,

where $p(\boldsymbol{a})$ is the prior on the parameters and $p\left(\left\{\boldsymbol{x}_{i}\right\}\right)$ takes care of normalization. The combination of $p\left(\boldsymbol{x}_{i} \mid \boldsymbol{a}\right)$ on the right-hand side of Eq. (17) is the likelihood, while $p\left(\boldsymbol{a} \mid\left\{\boldsymbol{x}_{i}\right\}\right)$ is the posterior probability. Obviously, each of the probability distributions introduced above is normalized to unity in the available part of the corresponding space

$$
\begin{gathered}
\int_{-\infty}^{+\infty} \ldots \int_{-\infty}^{+\infty} p\left(\boldsymbol{a} \mid\left\{\boldsymbol{x}_{i}\right\}\right) \mathrm{d} a_{1} \ldots \mathrm{d} a_{n}=1 \\
\int_{-\infty}^{+\infty} \cdots \int_{-\infty}^{+\infty} p\left(\boldsymbol{x}_{i} \mid \boldsymbol{a}\right) \mathrm{d} x_{1} \ldots \mathrm{d} x_{n}=1 .
\end{gathered}
$$

Van der Marel et al. (2000) proposed to restrict the considerations to the velocity space $\left(\boldsymbol{x}_{i}=v_{i}\right)$. The probability $p\left(v_{i} \mid \boldsymbol{a}\right)$ was given by (15) with a more detailed formula for $p_{v}$ and the assumption that $\alpha(R) \approx$ const., which was found to be roughly consistent with the data. However, as we have seen in Fig. 13 the dependence of the probability $\alpha$ on radius is in general not negligible. The most natural way to take this fact into account is simply to consider the probability on the whole projected space with $\boldsymbol{x}_{i}=\left(\boldsymbol{R}_{i}, v_{i}\right)$

$$
\begin{aligned}
p\left(R_{i}, v_{i} \mid\left(\boldsymbol{a}_{\mathrm{d}}, \alpha_{\mathrm{p}}\right)\right) \mathrm{d} R \mathrm{~d} v & =\alpha_{\mathrm{p}} f\left(R_{i}, v_{i} \mid \boldsymbol{a}_{\mathrm{d}}\right) \mathrm{d} R \mathrm{~d} v \\
& +\left(1-\alpha_{\mathrm{p}}\right) \frac{R}{R_{\text {max }}^{2} v_{\max }} \mathrm{d} R \mathrm{~d} v,
\end{aligned}
$$

where $f(R, v)$ is the projected distribution function of halo particles, $\boldsymbol{a}_{\mathrm{d}}$ is a set of dynamical parameters and $\alpha_{\mathrm{p}}$ is an additional free parameter describing the probability that a particle found at
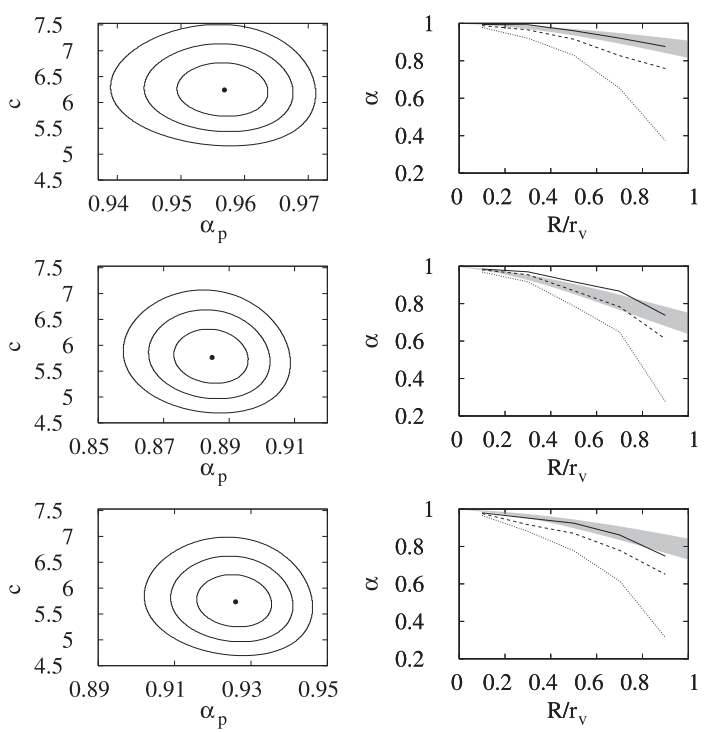

Fig. 14. Left column panels show the results of the Bayesian analysis in the form of contours in the $c-\alpha_{\mathrm{p}}$ parameter plane corresponding to 68.3, 95.4 and 99.73 percent confidence levels. In the right column we plot $\alpha(R)$ probability profiles calculated for different types of interlopers: particles beyond $r_{\mathrm{v}}$ (dotted lines) or $2 r_{\mathrm{v}}$ (dashed lines) and unbound particles (solid lines). Shaded areas indicate $\alpha(R)$ values corresponding to $c$ and $\alpha_{\mathrm{p}}$ parameters from the inside of the 99.73 percent probability contours. The rows of panels from top to bottom are for the $x, y$ and $z$ projections respectively.

any radius and with any velocity is a halo particle. The last term in (20) describes the uniform distribution of interlopers both in position on the sky and in velocity space.

We performed the analysis of our three stacked haloes using the probability distribution (20) with $f\left(R_{i}, v_{i} \mid \boldsymbol{a}\right)$ given for simplicity by the formula (12) parametrized by the $c$ parameter. Since we are dealing with stacked haloes and we assume isotropic orbits (as shown to be the case for early-type galaxies in Coma and other clusters, see Łokas \& Mamon 2003; Biviano $\&$ Katgert 2004), the only free parameters in this analysis are the concentration and the probability $\alpha_{\mathrm{p}}$. Results of this analysis are shown in the left column of Fig. 14 in the form of contours corresponding to $68.3,95.4$ and 99.73 percent confidence levels drawn in the $c-\alpha_{\mathrm{p}}$ parameter plane.

As we can see from Fig. 14, the preferred values of $c$ lie slightly below the value of the concentration parameter (estimated from the full 3D data) for the composite cluster, $c=6.9$, which is probably due to our simplistic probability distribution $f(R, v)$. However, we believe that the results concerning $\alpha_{\mathrm{p}}$ illustrate well the general features of interloper treatment in this approach since the distribution of interlopers used in formula (20) is independent of the dynamical model.

It is interesting to compare $\alpha_{\mathrm{p}}$ with abundances of different types of particles seen on velocity diagrams and the mean value of $\alpha$ obtained in the previous subsection. All these values are listed in Table 2, where $F_{<v_{\text {esc }}}$ and $F_{<2 r_{\mathrm{v}}}$ are the abundances of bound particles and particles within $2 r_{\mathrm{v}}$ respectively. Comparing these results, we notice an excellent agreement between $\alpha_{\mathrm{p}}$ and $\bar{\alpha}$. This fact is understandable since $\alpha$ was estimated in radial bins with the same number of particles inside each of them. Consequently one expects that the mean value of $\alpha$ measures the probability that a particle randomly chosen from the velocity diagram is a halo particle which is exactly the same probability as described by $\alpha_{\mathrm{p}}$. We also find that $\alpha_{\mathrm{p}}$ is approximately equal 
Table 2. Probabilities $\left(\alpha_{\mathrm{p}}\right.$ and $\left.\bar{\alpha}\right)$ of finding a halo particle on the velocity diagram estimated via indirect methods of interloper treatment. $F_{<v_{\text {esc }}}$ and $F_{<2 r_{\mathrm{v}}}$ are fractions of bound particles and particles within $2 r_{\mathrm{v}}$ respectively.

\begin{tabular}{ccccc}
\hline \hline Halo projection & $\alpha_{\mathrm{p}}$ & $\bar{\alpha}$ & $F_{<v_{\text {esc }}}$ & $F_{<2 r_{\mathrm{v}}}$ \\
\hline$x$ & $0.957_{-0.018}^{+0.014}$ & 0.946 & 0.958 & 0.909 \\
$y$ & $0.885_{-0.027}^{+0.022}$ & 0.883 & 0.903 & 0.852 \\
$z$ & $0.926_{-0.024}^{+0.020}$ & 0.927 & 0.905 & 0.856 \\
\hline
\end{tabular}

to the abundance of bound particles. This means that in this approach mainly unbound particles contribute to the distribution of interlopers. Some departure from this rule can be seen for the $z$-axis projection. However, if we take into account that the velocity distribution of unbound particles in this case is slightly peaked close to $v \sim V_{\mathrm{v}}$ (see the bottom right panel of Fig. 11) the situation is clarified: some of these interlopers are identified as halo particles increasing $\alpha_{\mathrm{p}}$.

Although we used here only the global probability $\alpha_{\mathrm{p}}$ of finding a halo particle in the velocity diagram, it is easy to reproduce the $\alpha(R)$ profile. In this case Eq. (16) has its counterpart in the form

$\alpha(R)=\frac{\Sigma_{M}(R, c)}{\Sigma_{M}(R, c)+\Sigma_{i}}$,

where $\Sigma_{i}=$ const. is the surface density of interlopers related to $\alpha_{\mathrm{p}}$ by

$\alpha_{\mathrm{p}}=\frac{\int_{0}^{R_{\max }} 2 \pi R \Sigma_{M}(R, c) \mathrm{d} R}{\int_{0}^{R_{\max }} 2 \pi R \Sigma_{M}(R, c) \mathrm{d} R+\pi R_{\max }^{2} \Sigma_{i}}$.

Therefore given $\alpha_{\mathrm{p}}$ and $c$ one is able to calculate $\alpha(R)$ profile. The results are plotted in the right column panels of Fig. 14 in the form of shaded areas which indicate $\alpha(R)$ values calculated for $c$ and $\alpha_{\mathrm{p}}$ parameters from the inside of the probability contour corresponding to 99.73 percent confidence level. We can clearly see that the area available for $\alpha(R)$ includes a profile calculated for interlopers as unbound particles.

We therefore confirm the results of the previous subsection that the uniform distribution of interlopers in the velocity diagram is mainly reproduced by unbound particles, whereas most of bound particles from the outside of the virial sphere contribute to the distribution function of halo particles. We emphasize that only indirect methods are able to take into account the presence of low velocity interlopers in the central part of the velocity diagram. Consequently, they can potentially include all unbound particles in the interloper background, which is beyond the reach of any direct scheme. For instance, applying $v_{\max }(1)$ method, the most successful among direct ones, to the composite velocity diagrams we found that 21,16 and 44 percent of unbound particles (for $x, y$ and $z$ projection respectively) are not identified and remain in the final samples of halo members resulting in the contamination on the level of $0.9,1.7$ and 4.4 percent respectively.

\section{Discussion}

We have studied different approaches to the treatment of interlopers in the analysis of kinematic data for galaxy clusters. For the direct methods of interloper removal their efficiency was measured by simple parameters: the fraction of removed interlopers $f_{\mathrm{i}}$, the fraction of removed members $f_{\mathrm{g}}$ and the fraction of non-identified interlopers remaining in the final samples $f_{\mathrm{h}}$.
The values of these parameters obtained by avergaing over 30 velocity diagrams studied here are given in Table 1 . We can see that the highest $f_{\mathrm{i}}$ (73 percent for unbound particles) is reached by $v_{\max }(1)$ method originally proposed by den Hartog \& Katgert (1996) although all the other methods considered (except for $\left.v_{\max }(2)\right)$ have $f_{\mathrm{i}}$ on the level of 60 percent. The differences in mean $f_{\mathrm{i}}$ between the methods are small compared to the dispersion obtained by averaging the results over 30 velocity diagrams which is about 20-30 percent in the case of unbound particles. In all direct methods of interloper removal $f_{\mathrm{i}}$ equals approximately 40 percent and 20 percent respectively for particles beyond $2 r_{\mathrm{V}}$ and $r_{\mathrm{v}}$ so the methods are not efficient in removing them. This occurs because particles from the close surroundings of the virial region are significantly concentrated around the halo mean velocity and may in fact be good tracers of the halo potential. All the methods show rather low fractions of members misidentified as interlopers (below 2 percent for all types of interlopers with a dispersion of about 2 percent). Furthermore, final samples of halo members include on average $2-4$ percent of unbound particles with dispersion of around 5 percent.

One may wonder if this is the most reliable way to compare the different methods. Since our purpose is to maximize $f_{\mathrm{i}}$ and minimize $f_{\mathrm{g}}$ and $f_{\mathrm{h}}$ one could construct some combination of these parameters assigning them different weights; we have not done this in order not to obscure the picture. One can also ask whether removing 60 percent of interlopers by one method cannot be better than removing 70 percent by another when it comes to the final estimation of the cluster parameters. It could happen that a smaller number of removed interlopers could lead to better estimates of mass because the interlopers were actually those causing the largest bias while larger number of interlopers can in principle lead to more biased results because the less significant interlopers were removed. Fortunately, this is not the case, the interlopers removed by the different methods are mostly the same, the difference is usually in the border-line particles which do not significantly contribute to the bias. We have also verified that the final, cleaned samples depend very weakly on the initial cut-off in velocity: reducing the cut-off to $3000 \mathrm{~km} \mathrm{~s}^{-1}$ from $4000 \mathrm{~km} \mathrm{~s}^{-1}$ with respect to the cluster mean produces almost identical final samples differing by only $1-2$ particles in a few out of 30 velocity diagrams.

Apart from the quantitative measures presented in Table 1 the choice between different methods of interloper removal is largely a matter of subjective preference. One could try to judge the methods by how strong their underlying assumptions are but the methods rely on such different assumptions that it is difficult to compare them. Another possibility is to look at their convergence. Here again $v_{\max }(1)$ method is recommended: it always converges, contrary e.g. to second most effective method $M_{\mathrm{P}} / M_{\mathrm{VT}}$ based on the ratio of mass estimators where the procedure has to be stopped at a rather arbitrary point. Furthermore, as shown by van Haarlem et al. (1997), $v_{\max }(1)$ approach leads to the data sample which reproduces real velocity dispersion better than the one obtained after applying $3 \sigma_{\text {los }}$ algorithm in the original form proposed by Yahil \& Vidal (1977). It is also worth noting that $v_{\max }(1)$ method does not involve any parameters or characteristic scales which could restrict its application to cluster-size objects. It has been recently successfully applied to tidally stripped dwarf spheroidal galaxies where it allows to clean the stellar kinematic samples from interlopers originating from the tidal tails and the Milky Way (Klimentowski et al. 2006).

An ultimate verification of the methods would of course come from the dynamical modelling performed on the cleaned 
samples. Here, however, other issues come into play: the quality of the final results of the modelling depends also on the actual method used and particular properties of an object (whether it is in equilibrium, whether it is spherically symmetric, how well is the kinematics sampled). These sources of error may have more impact on the final result than the differences between the methods of interloper removal studied here (see the recent study by Biviano et al. (2006) who have analyzed, after interloper removal with $v_{\max }(1)$ method, different observational effects affecting the determination of cluster mass and velocity dispersion). However, if interlopers were not removed at all the result would be very strongly biased and the contamination could become the main source of error. In any case it would be very difficult to disentangle the effect of interlopers from other sources of uncertainties.

Comparison between many modelling approaches possible is beyond the scope of this paper and it was not our purpose to provide here such a final answer. For the study of the performance of one of the dynamical analysis methods based on fitting the velocity dispersion and kurtosis profiles for clusters we refer the reader to Sanchis et al. (2004) and Łokas et al. (2006). Examples of such full dynamical modelling, including the interloper removal as an important first step, in the case of Abell 576 and other clusters with significant background contamination can be found in Wojtak \& Łokas (2006).

For the purpose of the present study we performed a simple test taking the cleaned particle samples for our 30 velocity diagrams obtained with $v_{\max }(1)$ method and fitting the velocity dispersion profiles to the solutions of the Jeans equation assuming isotropic orbits and estimating the virial masses and concentrations. Averaging over 30 diagrams we find that the ratio of the estimated virial mass to the real one measured from the $3 \mathrm{D}$ information is $M_{\mathrm{v}} / M_{\mathrm{v}, \text { true }}=0.86 \pm 0.23$ while for concentrations we get $c / c_{\text {true }}=1.76 \pm 1.01$. Interestingly, for the samples of bound particles the results are very similar: $M_{\mathrm{v}} / M_{\mathrm{v}, \text { true }}=0.85 \pm 0.18, c / c_{\text {true }}=1.91 \pm 1.31$. These values should be compared to the parameters obtained for the samples of all particles (without application of any interloper removal scheme): $M_{\mathrm{v}} / M_{\mathrm{v}, \text { true }}=1.64 \pm 0.92, c / c_{\text {true }}=0.34 \pm 0.31$. We therefore conclude that using the samples cleaned with $v_{\max }(1)$ method is equivalent to working with only bound particles. The significant bias still present in both cases, especially for concentration, can be explained by departure from isotropy of particle orbits in the simulated haloes which should be taken into account in more complete modelling.

We have also considered indirect methods of interloper treatment where their presence is accounted for in a statistical way. Here we have found that, contrary to the assumptions of van der Marel et al. (2001), the probability of a given galaxy being an interloper is not independent of the projected radius but increases with it. We have also verified the applicability of the approach of fitting a Gaussian plus a constant to the velocity distribution in galaxy clusters as a method to account for interlopers in estimating the velocity dispersion profile originally proposed by Prada et al. (2003) to study the velocity distribution of satellites around giant galaxies.

The main disadvantage of the indirect methods is that in order to reliably estimate the parameters they require large kinematic samples which can only be obtained by stacking data coming from many objects. This procedure, although commonly used, can be dangerous, because it is not clear how the distances and velocities should be scaled. While in the case of distances the choice of the virial radius as the scaling parameter seems rather obvious, in the case of velocities it is less clear whether one should use velocity dispersion, circular velocity at the virial radius or perhaps the maximum circular velocity and still all would be subject to uncertainties due to modelling of single clusters. In addition, the underlying assumption of indirect methods is that the background of interlopers is uniform which is never exactly the case due to clustering.

Acknowledgements. We wish to thank our referee, A. Biviano, for constructive comments which helped improve the paper. Computer simulations used in this work were performed at the Leibnizrechenzentrum (LRZ) in Munich. RW and EŁ are grateful for the hospitality of Astrophysikalisches Institut Potsdam, Institut d'Astrophysique de Paris and Instituto de Astrofísica de Andalucia where part of this work was done. RW acknowledges the summer student program at Copernicus Center. This work was partially supported by the Polish Ministry of Scientific Research and Information Technology under grant 1P03D02726 as well as the Jumelage program Astronomie France Pologne of CNRS/PAN, the Polish-Spanish exchange program of CSIC/PAN and the Polish-German exchange program of Deutsche Forschungsgemeinschaft (DFG).

\section{References}

Bahcall, J. N, \& Tremaine, S. 1981, ApJ, 244, 805 Bartelmann, M. 1996, A\&A, 313, 697

Beers, T. C., Geller, M. J., \& Huchra, J. P. 1982, ApJ, 257, 23

Binney, J., \& Mamon, G. A. 1982, MNRAS, 200, 361

Biviano, A., \& Girardi, M. 2003, ApJ, 585, 205

Biviano, A., \& Katgert, P. 2004, A\&A, 424, 779

Biviano, A., Murante, G., Borgani, S., et al. 2006, A\&A, 456, 23

Carlberg, R. G., Yee, H. K. C., Ellingson, E., et al. 1997, ApJ, 485, L13

Cen, R. 1997, ApJ, 485, 39

Chen, J., Kravtsov, A. V., Prada, F., et al. 2006, ApJ, 647, 86

den Hartog, R., \& Katgert, P. 1996, MNRAS, 279, 349

Diaferio, A. 1999, MNRAS, 309, 610

Diaferio, A., \& Geller, M. J. 1997, ApJ, 481, 633

Diaferio, A., Kauffmann, G., Colberg, J. M., White, S. D. M. 1999, MNRAS, 307,537

Diemand, J., Moore, B., \& Stadel, J. 2004, MNRAS, 352, 535

Fadda, D., Girardi, M., Giuricin, G., Mardirossian, F., \& Mezzetti, M. 1996, ApJ, 473,670

Faltenbacher, A., \& Diemand, J. 2006, MNRAS, 369, 1698

Fasano, G., Marmo, C., Varela, J., et al. 2006, A\&A, 445, 805

Hansen, S. H., Moore, B., Zemp, M., \& Stadel, J. 2006, JCAP, 1, 14

Heisler, J., Tremaine, S., \& Bahcall, J. N. 1985, ApJ, 298, 8

Katgert, P., Mazure, A., Perea, J., et al. 1996, A\&A, 310, 8

Kazantzidis, S., Magorrian, J., \& Moore, B. 2004, ApJ, 601, 37

Klimentowski, J., Łokas, E. L., Kazantzidis, S., et al. 2006, MNRAS, submitted, [arXiv:astro-ph/0611296]

Klypin, A., Hoffman, Y., Kravtsov, A. V., \& Gottlöber, S. 2003, ApJ, 596, 19

Kravtsov, A. V., Klypin, A. A., \& Khokhlov, A. M. 1997, ApJS, 111, 73

Limber, D. N., \& Mathews, W. G., 1960, ApJ, 132, 286

Łokas, E. L., \& Hoffman, Y. 2001, in Proc. 3rd International Workshop, The Identification of Dark Matter. ed. N. J. C. Spooner, V. Kudryavtsev (Singapore: World Scientific), 121

Łokas, E. L., \& Mamon, G. A. 2001, MNRAS, 321, 155

Łokas, E. L., \& Mamon, G. A. 2003, MNRAS, 343, 401

Łokas, E. L., Wojtak, R., Gottlöber, S., Mamon, G. A., \& Prada, F. 2006, MNRAS, 367, 1463

Mahdavi, A., \& Geller, M. J. 2004, ApJ, 607, 202

Mamon, G. A., \& Łokas, E. L. 2005, MNRAS, 363, 705

Maoz, E., \& Bekenstein, J. D. 1990, ApJ, 353, 59

Navarro, J. F., Frenk, C. S., \& White, S. D. M. 1997, ApJ, 490, 493

Perea, J., del Olmo, A., \& Moles, M., A\&A 1990, 237, 319

Prada, F., Vitvitska, M., Klypin, A., et al. 2003, ApJ, 598, 260

Prada, F., Klypin, A. A., Simonneau, et al. 2006, ApJ, 645, 1001

Prugniel, P., \& Simien, F. 1997, A\&A, 321, 111

Sanchis, T., Łokas, E. L., \& Mamon, G. A. 2004, MNRAS, 347, 1198

The, L. S., \& White, S. D. M., 1986, AJ, 92, 1248

van der Marel, R. P., Magorrian, J., Carlberg, R. G., Yee, H. K. C., \& Ellingson, E. 2000, ApJ, 119, 2038

van Haarlem, M. P., Frenk, C. S., \& White, S. D. M. 1997, MNRAS, 287, 817

Widrow, L. M. 2000, ApJS, 131, 39

Wojtak, R., \& Łokas, E. L. 2006, MNRAS, in press,

[arXiv: astro-ph/0606618]

Wojtak, R., Łokas, E. L., Gottlöber, S., \& Mamon, G. A. 2005, MNRAS, 361, L1

Yahil, A., \& Vidal, N. V. 1977, ApJ, 214, 347

Zabludoff, A. I., Huchra, J. P., \& Geller, M. J. 1990, ApJS, 74, 1 